\title{
Migration-driven benefit in a two-species nutrient taxis system
}

\author{
Piotr Krzyżanowski* $\quad$ Michael Winkler ${ }^{\dagger} \quad$ Dariusz Wrzosek*
}

\begin{abstract}
A model describing the competition of two species for a common nutrient is studied. It is assumed that one of the competitors is motionless while the other has the ability to move upwards gradients of the nutrient density. It is proved that under suitable assumptions on the initial data, in the long time perspective the ability to move turns out to be a crucial feature providing competitive advantage irrespectively of a possible difference between the species with regard to their rates of proliferation and nutrient uptake.
\end{abstract}

Key words: competition for common resources, chemotaxis, weak solutions

AMS: 35K57, 35K59, 35Q92, 35B40 (primary); 92C17, 35K65 (secondary)

\section{Introduction}

We consider a basic simplistic model describing the competition between two species feeding on a common single non-renewable resource - a nutrient which is indispensable for reproduction albeit not necessary to survive. Our goal is to study possible benefits which may result from the ability to move in searching for food as a factor determining success in competition. Indeed we will see that in some cases this factor becomes crucial and the usual population parameters indicating competitive benefits become irrelevant.

In particular, letting $w=w(x, t)$ denote the nutrient concentration and $u=u(x, t)$ and $v=v(x, t)$ represent the population densities of two competing species distributed in a bounded domain $\Omega \subset \mathbb{R}^{n}$, $n \geq 1$, we shall consider the problem

$$
\begin{cases}u_{t}=D_{u} \Delta u-\chi \nabla \cdot(u \nabla w)+\delta u w, & x \in \Omega, t>0, \\ v_{t}=\alpha v w, & x \in \Omega, t>0, \\ w_{t}=D_{w} \Delta w-\beta u w-\gamma v w, & x \in \Omega, t>0, \\ \frac{\partial u}{\partial \nu}=\frac{\partial w}{\partial \nu}=0, & x \in \partial \Omega, t>0, \\ u(x, 0)=u_{0}(x), \quad v(x, 0)=v_{0}(x), \quad w(x, 0)=w_{0}(x), & x \in \Omega,\end{cases}
$$

where $D_{u}>0$ and $D_{w}>0$ are diffusion constants and $\chi>0$ is the chemotactic sensitivity coefficient. The coefficient $\beta$ describes the consumption rate of the first species while $\delta$ determines the species specific rate of proliferation and similar interpretation applies to the second species.

\footnotetext{
*Institute of Applied Mathematics and Mechanics, Warsaw University, ul. Banacha 2, 02-097 Warszawa, Poland (d.wrzosek@mimuw.edu.pl, p.krzyzanowski@mimuw.edu.pl)

${ }^{\dagger}$ Institut für Mathematik, Universität Paderborn, 33098 Paderborn, Germany (michael.winkler@math.uni-paderborn.de) Funding: The work of this author was supported by Deutsche Forschungsgemeinschaft in the context of the project Analysis of chemptactic cross-diffusion in complex frameworks.
} 
Focusing on the interplay between population growth on one hand, and mobility enhancing effectiveness of foraging on the other hand we take into account neither mortality nor nutrient renewal which are usually considered in the class of models describing the competition for common resources. We underline that neglecting mortality terms in the model can be justified through a restriction to not too long time scales in the case of bacteria which after all proliferate by cell division. It should moreover be noted that in some related systems studied in the literature the rate of consumption is modelled by Monod-type functions accounting for effects of saturation which inevitably occur at higher food densities; as in our case the nutrient is being continuously depleted it will never reach higher levels of density at which any such saturation effect could become significant. For simplicity in presentation, we may therefore we restrict to bilinear Lotka-Volterra interaction noticing that our analysis can be extended to cases of widely arbitrary, and in particular to bounded, functional consumption rates.

The ODE part of the above system refers to model B studied in [6] in which logistic growth of the nutrient and mortality of competing species are assumed to be negligible in the time scale of our interest where the impact of species motility on feeding effectiveness is taken into account. The case of renewable resources and mortality in competing species demands a separate study. We refer a reader to Tilman's monograph [13] which contain basic ODE models of species competition for common resources particularly being related to experiments in a flow reactor named chemostat, also known as bio-reactor.

The classical ODE model for chemostat dynamics with constant supply of nutrients and stirring was analyzed in [6] where the long time behavior was described and especially competitive exclusion shown. Next, the corresponding reaction-diffusion system additionally accounting for random diffusion in an unstirred chemostat was studied e.g. in [7] and [4]. The role of chemotaxis in the process of nutrient competition was pointed out in the pioneering work [3], and then the resulting competition system with nutrient taxis in the spatially one-dimensional case in [15] and in [5], where inter alia a global attractor has been shown to exist.

It can be easily proved (see Appendix A) that in the case of the migration-free ODE system associated with (1.1), and hence also for the PDE system (1.1) with constant initial data, each individual trajectory $(u, v, w)$ approaches an equilibrium of the form $\left(u_{\infty}, v_{\infty}, 0\right)$ with some nonnegative constants $u_{\infty}$ and $v_{\infty}$; moreover, if the initial densities of both competitors are equal in that $u_{0}=v_{0}$, then the population with the higher proliferation coefficient asymptotically dominates in the sense that

$$
\operatorname{sgn}\left(u_{\infty}-v_{\infty}\right)=\operatorname{sgn}(\delta-\alpha) .
$$

The main intention of this work is to provide some analytical evidence indicating a substantial change of this picture when one of the competitors has the ability to move randomly and chemotactically towards increasing nutrient concentrations, whereas the other one remains sessile. This phenomenon is illustrated by numerical simulations which show the complexity of possible solutions behaviour depending on initial data and model parameters. Motility-driven beneficial effects in competitive biological systems were detected experimentally e.g. in the case of bacterial soilborne plant pathogen ([18], [10]); however, we are not aware of any analytical study rigorously confirming the occurrence of phenomena of this form in (1.1) or any related model.

Main results. In order to formulate our main results in this direction in a conveniently simple framework, let us firstly rescale (1.1) by noting that letting $\tilde{x}=\frac{x}{L}, \tilde{t}=\frac{t}{\tau}, u=U \tilde{u}, v=V \tilde{v}$ and $w=W \tilde{w}$ 
as well as $D=\frac{D_{u} \tau}{L^{2}}, 1=D_{w}^{\prime}=\frac{D_{w} W \tau}{L^{2}}=\delta^{\prime}=\delta \tau W, \chi^{\prime}=\frac{\chi U W \tau}{L^{2}}, \alpha^{\prime}=\alpha^{\prime} \tau W, \beta^{\prime}=\beta \tau U$ and $\gamma^{\prime}=\gamma \tau U$ for suitably chosen positive numbers $L, \tau, U, V$ and $W$, on performing standard computations and dropping tildes and primes we arrive at the normalized version of (1.3) given by

$$
\begin{cases}u_{t}=D \Delta u-\chi \nabla \cdot(u \nabla w)+u w, & x \in \Omega, t>0, \\ v_{t}=\alpha v w, & x \in \Omega, t>0, \\ w_{t}=\Delta w-\beta u w-\gamma v w, & x \in \Omega, t>0, \\ \frac{\partial u}{\partial \nu}=\frac{\partial w}{\partial \nu}=0, & x \in \partial \Omega, t>0, \\ u(x, 0)=u_{0}(x), \quad v(x, 0)=v_{0}(x), \quad w(x, 0)=w_{0}(x), & x \in \Omega,\end{cases}
$$

which will be the particular objective of our subsequent considerations. Here for the initial data, throughout the sequel we will suppose that their first two components are such that

$$
\left\{\begin{array}{l}
u_{0} \in C^{0} \text { with } u_{0}>0 \text { in } \bar{\Omega} \text { and } \\
v_{0} \in W^{2, \infty}(\Omega) \text { satisfies } v_{0}>0 \text { in } \bar{\Omega}
\end{array}\right.
$$

and that for the signal density we have

$$
w_{0} \in W^{1, \infty}(\Omega) \text { with } w_{0}>0 \text { in } \bar{\Omega} .
$$

Constituting an apparently necessary prerequisite for any qualitative analysis, the first of our results then asserts global solvability within a natural weak solution concept.

Theorem 1.1 Let $n \leq 5$ and $\Omega \subset \mathbb{R}^{n}$ be a bounded convex domain with smooth boundary, and suppose that $D, \chi, \alpha, \beta$ and $\gamma$ are positive. Then for any choice of $u_{0}, v_{0}$ and $w_{0}$ fulfilling (1.4) and (1.5), there exist nonnegative functions

$$
\left\{\begin{array}{l}
u \in L_{l o c}^{\frac{n+2}{n}}(\bar{\Omega} \times[0, \infty)) \cap L_{l o c}^{\frac{n+2}{n+1}}\left([0, \infty) ; W^{1, \frac{n+2}{n+1}}(\Omega)\right), \\
v \in L^{\infty}(\Omega \times(0, \infty)) \quad \text { and } \\
w \in L_{l o c}^{4}\left([0, \infty) ; W^{1,4}(\Omega)\right)
\end{array}\right.
$$

such that $(u, v, w)$ is a global weak solution of (1.3) in the sense of Definition 2.1.

We remark that in the case $n \leq 2$, by means of an adequate extension of the argument given in [11] (cf. also the outline in [17, Section 7]) it is possible to show that the a priori estimates derived in Section 2 below are actually sufficient to ensure global solvability even in the context of classical solutions. Since our focus will rather be on a qualitative analysis of (1.3), however, we refrain from pursuing this any further here.

Now the core of this study, to be addressed in Section 3, reveals that relative to the second subpopulation, the first indeed may eventually take advantage of its ability to migrate, as becoming manifest in our main result on qualitative behavior in (1.3):

Theorem 1.2 Let $n \leq 5, D>0, \chi>0, \alpha>0, \beta>0$ and $\gamma>0$, and suppose that $u_{0}$ and $v_{0}$ are such that (1.4) holds with $u_{0} \not \equiv$ const.. Then there exist $C>0$ and $T>0$ such that for all $M>0$ one can find $\sigma>0$ with the property that whenever $w_{0}$ complies with (1.5) and moreover satisfies

$$
\int_{\Omega} \frac{\left|\nabla w_{0}\right|^{2}}{w_{0}} \leq M
$$


and

$$
\left\|w_{0}\right\|_{L^{\infty}(\Omega)} \leq \sigma
$$

for the global weak solution $(u, v, w)$ of (1.3) from Theorem 1.1 we have $\ln \frac{v(\cdot, t)}{u(\cdot, t)} \in L^{1}(\Omega)$ for a.e. $t>T$ and

$$
I(t):=\int_{\Omega} \ln \frac{v(x, t)}{u(x, t)} d x \leq \int_{\Omega} \ln \frac{v_{0}}{u_{0}}-C \quad \text { for a.e. } t>T .
$$

Particularly, in the special case of precisely identical initial densities $u_{0}$ and $v_{0}$, not necessarily spatially constant, (1.9) states that for suitably small initial nutrient distributions, the first population ultimately prevails in the sense that then the corresponding logarithmic averages satisfy $\frac{1}{|\Omega|} \int_{\Omega} \ln u \geq \frac{1}{|\Omega|} \int_{\Omega} \ln v+C$ for all sufficiently large times, with a fixed positive constant $C$. We emphasize that in stark contrast to the migration-free asymptotics characterized by (1.2), through its qualitative independence of the sizes of $\alpha, \beta$ and $\gamma$ this result especially covers arbitrarily large values of $\alpha$ and hence applies to situations in which the static species, thus equipped with a substantial advantage in the efficiency of proliferation, might be expected to retain certain benefits thereof.

Section 4 finally contains results of numerical simulations performed in the case $n=1$, and for some radially symmetric solutions in three-dimensional balls. Going partially beyond situations addressed in Theorem 1.2, these findings will indicate that in general situations not necessarily complying with the hypotheses from Theorem 1.2, the final result of competition may heavily depend on a subtle interplay of many factors, including the initial distribution of species and nutrient, while the increase of space dimension seems to affect formation of more spiky space-time patterns.

\section{Regularization, a quasi-energy property and global solvability}

\subsection{A weak solution concept and a family of approximate problems}

In our analysis we shall pursue the following rather natural concept generalizing the notion of solution to (1.3).

Definition 2.1 Suppose that $u \in L_{l o c}^{1}\left([0, \infty) ; W^{1,1}(\Omega)\right), v \in L_{l o c}^{1}(\bar{\Omega} \times[0, \infty))$ and $w \in L_{l o c}^{\infty}(\bar{\Omega} \times[0, \infty)) \cap$ $L_{l o c}^{1}\left([0, \infty) ; W^{1,1}(\Omega)\right)$ are all nonnegative and such that $u \nabla w \in L_{l o c}^{1}\left(\bar{\Omega} \times[0, \infty) ; \mathbb{R}^{n}\right)$. Then $(u, v, w)$ will be called a global weak solution of (1.3) if for all $\varphi \in C_{0}^{\infty}(\bar{\Omega} \times[0, \infty))$, the identities

$$
-\int_{0}^{\infty} \int_{\Omega} u \varphi_{t}-\int_{\Omega} u_{0} \varphi(\cdot, 0)=-D \int_{0}^{\infty} \int_{\Omega} \nabla u \cdot \nabla \varphi+\chi \int_{0}^{\infty} \int_{\Omega} u \nabla w \cdot \nabla \varphi+\int_{0}^{\infty} \int_{\Omega} u w \varphi
$$

and

$$
-\int_{0}^{\infty} \int_{\Omega} v \varphi_{t}-\int_{\Omega} v_{0} \varphi(\cdot, 0)=\alpha \int_{0}^{\infty} \int_{\Omega} v w \varphi
$$

as well as

$$
-\int_{0}^{\infty} \int_{\Omega} w \varphi_{t}-\int_{\Omega} w_{0} \varphi(\cdot, 0)=-\int_{0}^{\infty} \int_{\Omega} \nabla w \cdot \nabla \varphi-\beta \int_{0}^{\infty} \int_{\Omega} u w \varphi-\gamma \int_{0}^{\infty} \int_{\Omega} v w \varphi
$$

are satisfied. 
In order to construct such a global weak solutions by means of a suitable approximation procedure, following [17] we fix a family $\left(F_{\varepsilon}\right)_{\varepsilon \in(0,1)} \subset C^{\infty}([0, \infty))$ of functions such that whenever $\varepsilon \in(0,1)$, we have

$$
F_{\varepsilon}(0)=0 \quad \text { and } \quad 0 \leq F_{\varepsilon}^{\prime}(s) \leq 1 \text { for all } s \geq 0,
$$

that $F_{\varepsilon}$ and $0 \leq s \mapsto s F_{\varepsilon}^{\prime}(s)$ are bounded on $[0, \infty)$ for each $\varepsilon \in(0,1)$, with $F_{\varepsilon}^{\prime}(s) \nearrow 1$ as $\varepsilon \searrow 0$ for all $s \geq 0$, noting that these requirements are met if e.g. we define

$$
F_{\varepsilon}(s):=\frac{s}{1+\varepsilon s} \quad \text { for } \varepsilon \in(0,1) \text { and } s \geq 0 .
$$

For $\varepsilon \in(0,1)$, we then consider

$$
\begin{cases}u_{\varepsilon t}=D \Delta u_{\varepsilon}-\chi \nabla \cdot\left(u_{\varepsilon} F_{\varepsilon}^{\prime}\left(u_{\varepsilon}\right) \nabla w_{\varepsilon}\right)+F_{\varepsilon}\left(u_{\varepsilon}\right) w_{\varepsilon}, & x \in \Omega, t>0, \\ v_{\varepsilon t}=\alpha v_{\varepsilon} w_{\varepsilon}, & x \in \Omega, t>0, \\ w_{\varepsilon t}=\Delta w_{\varepsilon}-\beta F_{\varepsilon}\left(u_{\varepsilon}\right) w_{\varepsilon}-\gamma v_{\varepsilon} w_{\varepsilon}, & x \in \Omega, t>0, \\ \frac{\partial u_{\varepsilon}}{\partial \nu}=\frac{\partial w_{\varepsilon}}{\partial \nu}=0, & x \in \partial \Omega, t>0, \\ u_{\varepsilon}(x, 0)=u_{0}(x), \quad v_{\varepsilon}(x, 0)=v_{0}(x), \quad w_{\varepsilon}(x, 0)=w_{0}(x), & x \in \Omega,\end{cases}
$$

By adapting essentially well-known arguments, as detailed e.g. in 12 for a related problem and thus omitted here, one can readily verify that all these problems admit globally defined classical solutions:

Lemma 2.1 Assume (1.4) and (1.5). Then for each $\varepsilon \in(0,1)$, there exist functions

$$
\left\{\begin{array}{l}
u_{\varepsilon} \in C^{0}(\bar{\Omega} \times[0, \infty)) \cap C^{2,1}(\bar{\Omega} \times(0, \infty)), \\
v_{\varepsilon} \in C^{1}(\bar{\Omega} \times[0, \infty)), \\
w_{\varepsilon} \in \bigcap_{p>n} C^{0}\left([0, \infty) ; W^{1, p}(\Omega)\right) \cap C^{2,1}(\bar{\Omega} \times(0, \infty)),
\end{array}\right.
$$

all positive in $\bar{\Omega} \times[0, \infty)$, such that $\left(u_{\varepsilon}, v_{\varepsilon}, w_{\varepsilon}\right)$ solves (2.5) in the classical sense in $\Omega \times(0, \infty)$.

The following bound for the total mass in the first solution component is elementary but important.

Lemma 2.2 Assume (1.4) and (1.5). Then for all $\varepsilon \in(0,1)$,

$$
\int_{\Omega} u_{\varepsilon}(\cdot, t) \leq \int_{\Omega} u_{0}+\frac{1}{\beta} \int_{\Omega} w_{0} \quad \text { for all } t>0
$$

Proof.We integrate the first and the third equation from (2.5) over $x \in \Omega$ to see that

$$
\frac{d}{d t} \int_{\Omega} u_{\varepsilon}=\int_{\Omega} F_{\varepsilon}\left(u_{\varepsilon}\right) w_{\varepsilon} \quad \text { for all } t>0
$$

and

$$
\frac{d}{d t} \int_{\Omega} w_{\varepsilon}=-\beta \int_{\Omega} F_{\varepsilon}\left(u_{\varepsilon}\right) w_{\varepsilon}-\gamma \int_{\Omega} v_{\varepsilon} w_{\varepsilon} \leq-\beta \int_{\Omega} F_{\varepsilon}\left(u_{\varepsilon}\right) w_{\varepsilon} \quad \text { for all } t>0 .
$$

Taking an appropriate linear combination and integrating in time shows that

$$
\int_{\Omega} u_{\varepsilon}(\cdot, t)+\frac{1}{\beta} \int_{\Omega} w_{\varepsilon}(\cdot, t) \leq \int_{\Omega} u_{0}+\frac{1}{\beta} \int_{\Omega} w_{0} \quad \text { for all } t>0
$$


and thus implies (2.7).

In view of the comparatively simple structures of the second and third equations in (2.5), for the respective solution components some basic information can even be obtained in a pointwise sense:

Lemma 2.3 Suppose that (1.4) and (1.5) hold. Then for all $\varepsilon \in(0,1)$,

$$
\inf _{y \in \Omega} v_{0}(y) \leq v_{\varepsilon}(x, t) \leq\left\|v_{0}\right\|_{L^{\infty}(\Omega)} \cdot e^{\frac{\alpha}{\kappa}\left\|w_{0}\right\|_{L^{\infty}(\Omega)}} \quad \text { for all } x \in \Omega \text { and } t>0
$$

and

$$
\left\|w_{\varepsilon}(\cdot, t)\right\|_{L^{\infty}(\Omega)} \leq\left\|w_{0}\right\|_{L^{\infty}(\Omega)} e^{-\kappa t} \quad \text { for all } t>0
$$

where

$$
\kappa:=\gamma \cdot \inf _{y \in \Omega} v_{0}(y)>0 .
$$

Proof.Since $v_{\varepsilon t} \geq 0$ by (2.5), the left inequality in (2.8) is obvious. As therefore

$$
w_{\varepsilon t} \leq \Delta w_{\varepsilon}-\gamma v_{\varepsilon} w_{\varepsilon} \leq \Delta w_{\varepsilon}-\kappa w_{\varepsilon} \quad \text { in } \Omega \times(0, \infty)
$$

due to (2.4), we next obtain (2.9) from the maximum principle. Since $\kappa$ is positive, on integration of the second equation in (2.5) this in turn implies that

$$
\begin{aligned}
v_{\varepsilon}(x, t) & =v_{0}(x) \cdot e^{\alpha \int_{0}^{t} w_{\varepsilon}(x, s) d s} \\
& \leq\left\|v_{0}\right\|_{L^{\infty}(\Omega)} \cdot \exp \left\{\alpha \int_{0}^{t}\left\|w_{0}\right\|_{L^{\infty}(\Omega)} e^{-\kappa s} d s\right\} \\
& \leq\left\|v_{0}\right\|_{L^{\infty}(\Omega)} \cdot \exp \left\{\alpha\left\|w_{0}\right\|_{L^{\infty}(\Omega)} \cdot \frac{1-e^{-\kappa t}}{\kappa}\right\} \quad \text { for all } x \in \Omega \text { and } t>0
\end{aligned}
$$

and thus establishes also the right inequality in (2.8).

\subsection{Constructing a quasi-energy functional}

Now the core of both our existence theory as well as our subsequent qualitative analysis will be formed by the following observation on presence of a favorable global quasi-dissipative structure in (2.5).

Lemma 2.4 Let $K>0$. Then there exists $C(K)>0$ such that if (1.4) and (1.5) hold as well as

$$
\left\|w_{0}\right\|_{L^{\infty}(\Omega)} \leq K
$$

then for each $\varepsilon \in(0,1)$,

$$
\mathcal{F}_{\varepsilon}(t):=\beta \int_{\Omega} u_{\varepsilon} \ln u_{\varepsilon}+\frac{\gamma \chi}{2 \alpha} \int_{\Omega} \frac{\left|\nabla v_{\varepsilon}\right|^{2}}{v_{\varepsilon}}+\frac{\chi}{2} \int_{\Omega} \frac{\left|\nabla w_{\varepsilon}\right|^{2}}{w_{\varepsilon}}, \quad t \geq 0,
$$

and

$$
\mathcal{D}_{\varepsilon}(t):=\int_{\Omega} \frac{\left|\nabla u_{\varepsilon}\right|^{2}}{u_{\varepsilon}}+\int_{\Omega}\left|\Delta w_{\varepsilon}\right|^{2}+\int_{\Omega}\left|\nabla w_{\varepsilon}\right|^{4}, \quad t>0,
$$

have the property that for any $t>0$ we have

$$
\frac{d}{d t} \mathcal{F}_{\varepsilon}+\frac{1}{C(K)} \cdot \mathcal{D}_{\varepsilon}\left[u_{\varepsilon}(\cdot, t), w_{\varepsilon}(\cdot, t)\right] \leq C(K) e^{-\kappa t} \cdot\left\{1+\mathcal{F}_{\varepsilon}\left[u_{\varepsilon}(\cdot, t), v_{\varepsilon}(\cdot, t), w_{\varepsilon}(\cdot, t)\right]\right\},
$$

where $\kappa>0$ is taken from (2.10). 
Proof.By using (2.5), we compute

$$
\frac{d}{d t} \int_{\Omega} u_{\varepsilon} \ln u_{\varepsilon}=-D \int_{\Omega} \frac{\left|\nabla u_{\varepsilon}\right|^{2}}{u_{\varepsilon}}+\chi \int_{\Omega} F_{\varepsilon}^{\prime}\left(u_{\varepsilon}\right) \nabla u_{\varepsilon} \cdot \nabla w_{\varepsilon}+\int_{\Omega} F_{\varepsilon}\left(u_{\varepsilon}\right) \ln u_{\varepsilon} \cdot w_{\varepsilon}+\int_{\Omega} F_{\varepsilon}\left(u_{\varepsilon}\right) w_{\varepsilon}
$$

and

$$
\frac{d}{d t} \int_{\Omega} \frac{\left|\nabla v_{\varepsilon}\right|^{2}}{v_{\varepsilon}}=\alpha \int_{\Omega} \frac{w_{\varepsilon}}{v_{\varepsilon}}\left|\nabla v_{\varepsilon}\right|^{2}+2 \alpha \int_{\Omega} \nabla v_{\varepsilon} \cdot \nabla w_{\varepsilon}
$$

as well as

$$
\begin{aligned}
\frac{d}{d t} \int_{\Omega} \frac{\left|\nabla w_{\varepsilon}\right|^{2}}{w_{\varepsilon}}= & -\int_{\Omega} \frac{1}{w_{\varepsilon}} \Delta\left|\nabla w_{\varepsilon}\right|^{2}-2 \int_{\Omega} \frac{1}{w_{\varepsilon}}\left|D^{2} w_{\varepsilon}\right|^{2}-\int_{\Omega} \frac{\left|\nabla w_{\varepsilon}\right|^{2}}{w_{\varepsilon}^{2}} \Delta w_{\varepsilon} \\
& -\beta \int_{\Omega} \frac{F_{\varepsilon}\left(u_{\varepsilon}\right)}{w_{\varepsilon}}\left|\nabla w_{\varepsilon}\right|^{2}-2 \beta \int_{\Omega} F_{\varepsilon}^{\prime}\left(u_{\varepsilon}\right) \nabla u_{\varepsilon} \cdot \nabla w_{\varepsilon} \\
& -\gamma \int_{\Omega} \frac{v_{\varepsilon}}{w_{\varepsilon}}\left|\nabla w_{\varepsilon}\right|^{2}-2 \gamma \int_{\Omega} \nabla v_{\varepsilon} \cdot \nabla w_{\varepsilon}
\end{aligned}
$$

for all $t>0$. Here we recall that for all positive $\varphi \in C^{3}(\bar{\Omega})$ fulfilling $\frac{\partial \varphi}{\partial \nu}=0$ on $\partial \Omega$, by straightforward computation relying on the fact that then $\frac{\partial|\nabla \varphi|^{2}}{\partial \nu} \leq 0$ on $\partial \Omega$ by convexity of $\Omega([8])$,

$$
-\int_{\Omega} \frac{1}{\varphi} \Delta|\nabla \varphi|^{2}-2 \int_{\Omega} \frac{1}{\varphi}\left|D^{2} \varphi\right|^{2}-\int_{\Omega} \frac{|\nabla \varphi|^{2}}{\varphi^{2}} \Delta \varphi \leq-2 \int_{\Omega} w_{\varepsilon}\left|D^{2} \ln \varphi\right|^{2},
$$

and that there exist $c_{1}>0$ and $c_{2}>0$ with the property that for any such $\varphi$ we have

$$
\int_{\Omega} \frac{|\Delta \varphi|^{2}}{\varphi} \leq c_{1} \int_{\Omega} \varphi\left|D^{2} \ln \varphi\right|^{2}
$$

as well as

$$
\int_{\Omega} \frac{|\nabla \varphi|^{4}}{\varphi^{3}} \leq c_{2} \int_{\Omega} \varphi\left|D^{2} \ln \varphi\right|^{2}
$$


(cf. [16, Section 3]). Therefore, combining (2.15)-(2.17) shows that

$$
\begin{aligned}
\frac{d}{d t}\left\{\beta \int_{\Omega} u_{\varepsilon}\right. & \left.\ln u_{\varepsilon}+\frac{\gamma \chi}{2 \alpha} \int_{\Omega} \frac{\left|\nabla v_{\varepsilon}\right|^{2}}{v_{\varepsilon}}+\frac{\chi}{2} \int_{\Omega} \frac{\left|\nabla w_{\varepsilon}\right|^{2}}{w_{\varepsilon}}\right\} \\
\leq & -\beta D \int_{\Omega} \frac{\left|\nabla u_{\varepsilon}\right|^{2}}{u_{\varepsilon}}+\beta \chi \int_{\Omega} F_{\varepsilon}^{\prime}\left(u_{\varepsilon}\right) \nabla u_{\varepsilon} \cdot \nabla w_{\varepsilon}+\beta \int_{\Omega} F_{\varepsilon}\left(u_{\varepsilon}\right) \ln u_{\varepsilon} \cdot w_{\varepsilon}+\beta \int_{\Omega} F_{\varepsilon}\left(u_{\varepsilon}\right) w_{\varepsilon} \\
& +\frac{\gamma \chi}{2} \int_{\Omega} \frac{w_{\varepsilon}}{v_{\varepsilon}}\left|\nabla v_{\varepsilon}\right|^{2}+\gamma \chi \int_{\Omega} \nabla v_{\varepsilon} \cdot \nabla w_{\varepsilon} \\
& -\frac{\chi}{2} \int_{\Omega} w_{\varepsilon}\left|D^{2} \ln w_{\varepsilon}\right|^{2} \\
& -\frac{\beta \chi}{2} \int_{\Omega} \frac{F_{\varepsilon}\left(u_{\varepsilon}\right)}{w_{\varepsilon}}\left|\nabla w_{\varepsilon}\right|^{2}-\beta \chi \int_{\Omega} F_{\varepsilon}^{\prime}\left(u_{\varepsilon}\right) \nabla u_{\varepsilon} \cdot \nabla w_{\varepsilon} \\
& -\frac{\gamma \chi}{2} \int_{\Omega} \frac{v_{\varepsilon}}{w_{\varepsilon}}\left|\nabla w_{\varepsilon}\right|^{2}-\gamma \chi \int_{\Omega} \nabla v_{\varepsilon} \cdot \nabla w_{\varepsilon} \\
\leq & -\beta D \int_{\Omega} \frac{\left|\nabla u_{\varepsilon}\right|^{2}}{u_{\varepsilon}}-\frac{\chi}{2 c_{1}} \int_{\Omega} \frac{\left|\Delta w_{\varepsilon}\right|^{2}}{w_{\varepsilon}}-\frac{\chi}{2 c_{2}} \int_{\Omega} \frac{\left|\nabla w_{\varepsilon}\right|^{4}}{w_{\varepsilon}^{3}} \\
& +\frac{\gamma \chi}{2} \int_{\Omega} \frac{w_{\varepsilon}}{v_{\varepsilon}}\left|\nabla v_{\varepsilon}\right|^{2}+\beta \int_{\Omega} F_{\varepsilon}\left(u_{\varepsilon}\right) \ln u_{\varepsilon} \cdot w_{\varepsilon}+\beta \int_{\Omega} F_{\varepsilon}\left(u_{\varepsilon}\right) w_{\varepsilon} \quad \text { for all } t>0,
\end{aligned}
$$

where using that

$$
\left\|w_{\varepsilon}(\cdot, t)\right\|_{L^{\infty}(\Omega)} \leq K e^{-\kappa t} \leq K \quad \text { for all } t>0
$$

by (2.9) and (2.11), we can estimate

$$
\frac{\chi}{2 c_{1}} \int_{\Omega} \frac{\left|\Delta w_{\varepsilon}\right|^{2}}{w_{\varepsilon}} \geq \frac{\chi}{2 c_{1} K} \int_{\Omega}\left|\Delta w_{\varepsilon}\right|^{2} \quad \text { and } \quad \frac{\chi}{2 c_{2}} \int_{\Omega} \frac{\left|\nabla w_{\varepsilon}\right|^{4}}{w_{\varepsilon}^{3}} \geq \frac{\chi}{2 c_{2} K^{3}} \int_{\Omega}\left|\nabla w_{\varepsilon}\right|^{4}
$$

for all $t>0$. As from (2.19) and the validity of the inequality $z \ln z \geq-\frac{1}{e}$ for all $z>0$ we moreover obtain that

$$
\begin{aligned}
\frac{\gamma \chi}{2} \int_{\Omega} \frac{w_{\varepsilon}}{v_{\varepsilon}}\left|\nabla v_{\varepsilon}\right|^{2} & \leq \frac{\gamma \chi K}{2} e^{-\kappa t} \cdot \int_{\Omega} \frac{\left|\nabla v_{\varepsilon}\right|^{2}}{v_{\varepsilon}} \\
& =\alpha K e^{-\kappa t} \cdot\left\{\mathcal{F}_{\varepsilon}(t)-\beta \int_{\Omega} u_{\varepsilon} \ln u_{\varepsilon}-\frac{\chi}{2} \int_{\Omega} \frac{\left|\nabla w_{\varepsilon}\right|^{2}}{w_{\varepsilon}}\right\} \\
& \leq \alpha K e^{-\kappa t} \cdot \mathcal{F}_{\varepsilon}(t)-\alpha \beta K e^{-\kappa t} \cdot \int_{\left\{u_{\varepsilon}<1\right\}} u_{\varepsilon} \ln u_{\varepsilon} \\
& \leq \alpha K e^{-\kappa t} \cdot \mathcal{F}_{\varepsilon}(t)+\frac{\alpha \beta K|\Omega|}{e} \cdot e^{-\kappa t} \quad \text { for all } t>0
\end{aligned}
$$


and, similarly,

$$
\begin{aligned}
\beta \int_{\Omega} F_{\varepsilon}\left(u_{\varepsilon}\right) \ln u_{\varepsilon} \cdot w_{\varepsilon} & \leq \beta \int_{\left\{u_{\varepsilon}>1\right\}} F_{\varepsilon}\left(u_{\varepsilon}\right) \ln u_{\varepsilon} \cdot w_{\varepsilon} \\
& \leq \beta K e^{-\kappa t} \cdot \int_{\left\{u_{\varepsilon}>1\right\}} u_{\varepsilon} \ln u_{\varepsilon} \\
& =\beta K e^{-\kappa t} \cdot\left\{\int_{\Omega} u_{\varepsilon} \ln u_{\varepsilon}-\int_{\left\{u_{\varepsilon}<1\right\}} u_{\varepsilon} \ln u_{\varepsilon}\right\} \\
& \leq \beta K e^{-\kappa t} \cdot\left\{\int_{\Omega} u_{\varepsilon} \ln u_{\varepsilon}+\frac{|\Omega|}{e}\right\} \\
& \leq \beta K e^{-\kappa t} \cdot \mathcal{F}_{\varepsilon}(t)+\frac{\beta K|\Omega|}{e} e^{-\kappa t} \quad \text { for all } t>0
\end{aligned}
$$

as well as

$$
\beta \int_{\Omega} F_{\varepsilon}\left(u_{\varepsilon}\right) w_{\varepsilon} \leq \beta K e^{-\kappa t} \int_{\Omega} u_{\varepsilon} \leq \beta K \cdot\left\{\int_{\Omega} u_{0}+\frac{1}{\beta} \int_{\Omega} w_{0}\right\} \quad \text { for all } t>0
$$

due to (2.7), we readily infer that (2.18) entails (2.14) with some appropriately large $C(K)>0$.

\subsection{Resulting a priori estimates}

Since the factor $e^{-\kappa t}$ appearing on the right of (2.14) is integrable over $(0, \infty)$, our first conclusion from Lemma 2.4 asserts some space-time bounds for the quantities making up the dissipation rate $\mathcal{D}_{\varepsilon}$ therein even over this entire unbounded time interval.

Lemma 2.5 Assume that $u_{0}$ and $v_{0}$ comply with 1.4). Then for all $M>0$ and $K>0$ there exists $C(M, K)>0$ such that if $w_{0}$ satisfies (1.5) as well as (1.7) and (2.11), we have

$$
\int_{0}^{\infty} \int_{\Omega} \frac{\left|\nabla u_{\varepsilon}\right|^{2}}{u_{\varepsilon}} \leq C(M, K) \quad \text { for all } \varepsilon \in(0,1)
$$

and

as well as

$$
\int_{0}^{\infty} \int_{\Omega}\left|\Delta w_{\varepsilon}\right|^{2} \leq C(M, K) \quad \text { for all } \varepsilon \in(0,1)
$$

$$
\int_{0}^{\infty} \int_{\Omega}\left|\nabla w_{\varepsilon}\right|^{4} \leq C(M, K) \quad \text { for all } \varepsilon \in(0,1) .
$$

Proof.On invoking Lemma 2.4, we obtain $c_{1}=c_{1}(K)>0$ and $c_{2}=c_{2}(K)>0$ such that whenever $\varepsilon \in(0,1)$, with $\kappa>0$ as in (2.10) we have

$$
\frac{d}{d t} \mathcal{F}_{\varepsilon}(t)++c_{1} \mathcal{D}_{\varepsilon}(t) \leq c_{2} e^{-\kappa t} \cdot\left(1+\mathcal{F}_{\varepsilon}(t)\right) \quad \text { for all } t>0,
$$

from which on dropping the second summand on the left we infer by comparison that

$$
\begin{aligned}
\mathcal{F}_{\varepsilon}(t) & \leq \mathcal{F}_{\varepsilon}(0) e^{c_{2} \int_{0}^{t} e^{-\kappa s} d s}+\int_{0}^{t} e^{c_{2} \int_{s}^{t} e^{-\kappa \sigma} d \sigma} \cdot c_{2} e^{-\kappa s} d s \\
& \leq c_{3} \equiv c_{3}(M, K):=\left\{\beta \cdot\left|\int_{\Omega} u_{0} \ln u_{0}\right|+\frac{\gamma \chi}{2 \alpha} \int_{\Omega} \frac{\left|\nabla v_{0}\right|^{2}}{v_{0}}+\frac{\chi M}{2}\right\} \cdot e^{\frac{c_{2}}{\kappa}}+\frac{c_{2}}{\kappa} \cdot e^{\frac{c_{2}}{\kappa}} \quad \text { for all } t>0
\end{aligned}
$$


according to (1.7). Thereafter, an integration of (2.24) shows that

$$
\begin{aligned}
c_{1} \int_{0}^{t} \mathcal{D}_{\varepsilon}(s) d s & \leq \mathcal{F}_{\varepsilon}(0)-\mathcal{F}_{\varepsilon}(t)+c_{2} \int_{0}^{t} e^{-\kappa s} \cdot\left(1+\mathcal{F}_{\varepsilon}(s)\right) d s \\
& \leq c_{3}-\beta \int_{\Omega} u_{\varepsilon}(\cdot, t) \ln u_{\varepsilon}(\cdot, t)+\frac{c_{2} \cdot\left(1+c_{3}\right)}{\kappa} \\
& \leq c_{3}+\frac{\beta|\Omega|}{e}+\frac{c_{2} \cdot\left(1+c_{3}\right)}{\kappa}:=C(M, K)
\end{aligned}
$$

and thereby implies (2.21)-(2.23) in view of the definition (2.13) of $\mathcal{D}_{\varepsilon}$.

When restricted to bounded time intervals and combined with (2.7), by means of straightforward interpolation the above inequalities can be seen to entail some estimates also for $u_{\varepsilon}$ and $\nabla u_{\varepsilon}$ themselves, without appearance of any weight function.

Lemma 2.6 Assume (1.4), and let $M>0, K>0$ and $T>0$. Then there exists $C(M, K, T)>0$ with the property that whenever $w_{0}$ satisfies (1.5), (1.7) and (2.11), we have

$$
\int_{0}^{T} \int_{\Omega} u_{\varepsilon}^{\frac{n+2}{n}} \leq C(M, K, T) \quad \text { for all } \varepsilon \in(0,1)
$$

and

$$
\int_{0}^{T} \int_{\Omega}\left|\nabla u_{\varepsilon}\right|^{\frac{n+2}{n+1}} \leq C(M, K, T) \quad \text { for all } \varepsilon \in(0,1) .
$$

Proof.The estimate (2.25) follows by straightforward interpolation between the spatio-temporal $L^{2}$ estimate for $\nabla \sqrt{u_{\varepsilon}}$ from (2.21) and (2.7). Thereafter, (2.26) becomes a consequence of (2.25) and again (2.21) by means of the Hölder inequality (see also [17, Lemma 3.2]).

For the second component, on the basis of the ODE therefor in (2.5), as well as (2.23), we obtain the following.

Lemma 2.7 If (1.4) holds, then for all $M>0, K>0$ and $T>0$ one can find $C(M, K, T)>0$ such that if $w_{0}$ satisfies (1.5), (1.7) and (2.11), then for all $\varepsilon \in(0,1)$,

$$
\int_{\Omega}\left|\nabla v_{\varepsilon}(\cdot, t)\right|^{4} \leq C(M, K, T) \quad \text { for all } t \in(0, T) .
$$

Moreover,

$$
\left|v_{\varepsilon t}(x, t)\right| \leq \alpha K e^{\frac{\alpha K}{\kappa}}\left\|v_{0}\right\|_{L^{\infty}(\Omega)} \quad \text { for all } x \in \Omega, t>0 \text { and } \varepsilon \in(0,1),
$$

where $\kappa>0$ is as in (2.10).

Proof.We differentiate the identity

$$
v_{\varepsilon}(x, t)=v_{0}(x) e^{-\int_{0}^{t} \alpha w_{\varepsilon}(x, s) d s}, \quad x \in \Omega, t>0,
$$


and use (2.9) and (2.11) to estimate

$$
\begin{aligned}
\left|\nabla v_{\varepsilon}(x, t)\right| & =\left|\alpha v_{0}(x) e^{\alpha \int_{0}^{t} w_{\varepsilon}(x, s) d s} \cdot \int_{0}^{t} \nabla w_{\varepsilon}(x, s) d s+\nabla v_{0}(x) \cdot e^{\alpha \int_{0}^{t} w_{\varepsilon}(x, s) d s}\right| \\
& \leq \alpha\left\|v_{0}\right\|_{L^{\infty}(\Omega)} \cdot e^{\alpha K \int_{0}^{t} e^{-\kappa s} d s} \int_{0}^{t}\left|\nabla w_{\varepsilon}(x, s)\right| d s+\left\|\nabla v_{0}\right\|_{L^{\infty}(\Omega)} \cdot e^{\alpha K \int_{0}^{t} e^{-\kappa s} d s} \\
& \leq c_{1} \int_{0}^{t}\left|\nabla w_{\varepsilon}(x, s)\right| d s+c_{2} \quad \text { for all } x \in \Omega \text { and } t>0,
\end{aligned}
$$

where $c_{1}:=\alpha\left\|v_{0}\right\|_{L^{\infty}(\Omega)} \cdot e^{\frac{\alpha K}{\kappa}}$ and $c_{2}:=\left\|\nabla v_{0}\right\|_{L^{\infty}(\Omega)} \cdot e^{\frac{\alpha K}{\kappa}}$. After an integration over $\Omega$, according to the Hölder inequality this shows that

$$
\begin{aligned}
\int_{\Omega}\left|\nabla v_{\varepsilon}(x, t)\right|^{4} d x & \leq 8 c_{1}^{4} \int_{\Omega}\left\{\int_{0}^{t}\left|\nabla w_{\varepsilon}(x, s)\right| d s\right\}^{4} d x+8 c_{2}^{4} \\
& \leq 8 c_{1}^{4} \cdot \int_{\Omega}\left\{\int_{0}^{t}\left|\nabla w_{\varepsilon}(x, s)\right|^{4} d s\right\} \cdot t^{3} d x+8 c_{2}^{4} \\
& \leq 8 c_{1}^{4} T^{3} \cdot \int_{0}^{\infty} \int_{\Omega}\left|\nabla w_{\varepsilon}\right|^{4}+8 c_{2}^{4} \quad \text { for all } t>0
\end{aligned}
$$

and hence implies (2.27) due to (2.23).

The explicit inequality in (2.28) is an immediate consequence of (2.9), (2.11) and (2.8).

Our derivation of bounds for the time derivatives of $u_{\varepsilon}$ and $w_{\varepsilon}$ by means of Lemma 2.5 in the next two lemmata is rather straightforward.

Lemma 2.8 Assume (1.4). Then for all $M>0, K>0$ and $T>0$ there exists $C(M, K, T)>0$ with the property that whenever $w_{0}$ satisfies (1.5), (1.7) and (2.11), we have

$$
\int_{0}^{T}\left\|u_{\varepsilon t}(\cdot, t)\right\|_{\left(W^{1, p}(\Omega)\right)^{\star}}^{q} d t \leq C(M, K, T) \quad \text { for all } \varepsilon \in(0,1),
$$

where

$$
p:=\max \left\{n+2, \frac{4(n+2)}{6-n}\right\} \quad \text { and } \quad q:=\min \left\{\frac{n+2}{n+1}, \frac{4(n+2)}{5 n+2}\right\}>1 .
$$

Proof.Given $t>0$ and $\psi \in C^{1}(\bar{\Omega})$, using (2.5) along with (2.4) and the Hölder inequality we obtain

$$
\begin{aligned}
\left|\int_{\Omega} u_{\varepsilon t}(\cdot, t) \cdot \psi\right|= & \left|-D \int_{\Omega} \nabla u_{\varepsilon} \cdot \nabla \psi+\chi \int_{\Omega} u_{\varepsilon} F_{\varepsilon}^{\prime}\left(u_{\varepsilon}\right) \nabla w_{\varepsilon} \cdot \nabla \psi+\int_{\Omega} F_{\varepsilon}\left(u_{\varepsilon}\right) w_{\varepsilon} \psi\right| \\
\leq & D\left\|\nabla u_{\varepsilon}\right\|_{L^{\frac{n+2}{n+1}}(\Omega)}\|\nabla \psi\|_{L^{n+2}(\Omega)}+\chi\left\|u_{\varepsilon}\right\|_{L^{\frac{n+2}{n}}(\Omega)}\left\|\nabla w_{\varepsilon}\right\|_{L^{4}(\Omega)}\|\nabla \psi\|_{L^{\frac{4(n+2)}{6-n}}(\Omega)} \\
& +\left\|u_{\varepsilon}\right\|_{L^{\frac{n+2}{n}}(\Omega)}\left\|w_{\varepsilon}\right\|_{L^{\infty}(\Omega)}\|\psi\|_{L^{\frac{n+2}{2}}(\Omega)} \quad \text { for all } \varepsilon \in(0,1) .
\end{aligned}
$$

Since (2.30) warrants that $W^{1, p}(\Omega)$ is continuously embedded into $W^{1, n+2}(\Omega), W^{1, \frac{4(n+2)}{6-n}}(\Omega)$ and $L^{\frac{n+2}{2}}(\Omega)$, we thus infer the existence of $c_{1}>0$ such that

$$
\begin{aligned}
\left\|u_{\varepsilon t}(\cdot, t)\right\|_{\left(W^{1, p}(\Omega)\right)^{\star}} \leq & c_{1}\left\|\nabla u_{\varepsilon}\right\|_{L^{\frac{n+2}{n+1}}(\Omega)}+c_{1}\left\|u_{\varepsilon}\right\|_{L^{\frac{n+2}{n}}(\Omega)}\left\|\nabla w_{\varepsilon}\right\|_{L^{4}(\Omega)} \\
& +c_{1}\left\|u_{\varepsilon}\right\|_{L^{\frac{n+2}{n}}(\Omega)}\left\|w_{\varepsilon}\right\|_{L^{\infty}(\Omega)} \quad \text { for all } t>0 \text { and } \varepsilon \in(0,1),
\end{aligned}
$$


so that in view of Young's inequality, an integration yields

$$
\begin{aligned}
\int_{0}^{T}\left\|u_{\varepsilon t}(\cdot, t)\right\|_{\left(W^{1, p}(\Omega)\right)^{\star}}^{q} d t \leq & c_{1}^{q} \int_{0}^{T}\left\|\nabla u_{\varepsilon}(\cdot, t)\right\|_{L^{\frac{n+2}{n+1}(\Omega)}}^{q} d t+c_{1}^{q} \int_{0}^{T}\left\|u_{\varepsilon}(\cdot, t)\right\|_{L^{\frac{n+2}{n}}(\Omega)}^{q}\left\|\nabla w_{\varepsilon}(\cdot, t)\right\|_{L^{4}(\Omega)}^{q} d t \\
& +c_{1}^{q} \int_{0}^{T}\left\|u_{\varepsilon}(\cdot, t)\right\|_{L^{\frac{n+2}{n}}(\Omega)}^{q}\left\|w_{\varepsilon}(\cdot, t)\right\|_{L^{\infty}(\Omega)}^{q} d t \\
\leq & c_{1}^{q} \int_{0}^{T}\left\|\nabla u_{\varepsilon}(\cdot, t)\right\|_{L^{\frac{n+2}{n+1}}}^{\frac{n+2}{n+1}(\Omega)} d t+c_{1} T \\
& +c_{1}^{q} \int_{0}^{T}\left\|u_{\varepsilon}(\cdot, t)\right\|_{L^{\frac{n+2}{n}}(\Omega)}^{\frac{n+2}{n}} d t+c_{1}^{q} \int_{0}^{T}\left\|\nabla w_{\varepsilon}(\cdot, t)\right\|_{L^{4}(\Omega)}^{4} d t+c_{1} T \\
& +c_{1} \int_{0}^{T}\left\|u_{\varepsilon}(\cdot, t)\right\|_{L^{\frac{n+2}{n}}(\Omega)}^{\frac{n+2}{n}} d t+c_{1}^{q} \int_{0}^{T}\left\|w_{\varepsilon}(\cdot, t)\right\|_{L^{\infty}(\Omega)}^{\frac{n+2}{n+2}(\Omega)} d t
\end{aligned}
$$

for all $\varepsilon \in(0,1)$, because $q \leq \frac{n+2}{n+1}<\frac{n+2}{n}$ and $\frac{n}{n+2}+\frac{1}{4}+\frac{1}{q} \leq 1$ by (2.30). Therefore, (2.29) results from (2.26), (2.25), (2.23) and (2.9).

Lemma 2.9 Assume 1.4). Then for all $M>0, K>0$ and $T>0$ there exists $C(M, K, T)>0$ with the property that whenever $w_{0}$ satisfies (1.5), (1.7) and (2.11), we have

$$
\int_{0}^{T}\left\|w_{\varepsilon t}(\cdot, t)\right\|_{\left(W_{0}^{1, \infty}(\Omega)\right)^{\star}} d t \leq C(M, K, T) \quad \text { for all } \varepsilon \in(0,1) .
$$

Proof.We fix $\psi \in C_{0}^{\infty}(\Omega)$ such that $\|\psi\|_{W^{1, \infty}(\Omega)} \leq 1$ and then see using (2.5), (2.4) and Young's inequality that

$$
\begin{aligned}
\left|\int_{\Omega} w_{\varepsilon t} \cdot \psi\right| & =\left|-\int_{\Omega} \nabla w_{\varepsilon} \cdot \nabla \psi-\beta \int_{\Omega} F_{\varepsilon}\left(u_{\varepsilon}\right) w_{\varepsilon} \psi-\gamma \int_{\Omega} v_{\varepsilon} w_{\varepsilon} \psi\right| \\
& \leq \int_{\Omega}\left|\nabla w_{\varepsilon}\right|+\beta \int_{\Omega} u_{\varepsilon} w_{\varepsilon}+\gamma \int_{\Omega} v_{\varepsilon} w_{\varepsilon} \\
& \leq \int_{\Omega}\left|\nabla w_{\varepsilon}\right|^{4}+|\Omega|+\beta\left\|u_{\varepsilon}\right\|_{L^{1}(\Omega)}\left\|w_{\varepsilon}\right\|_{L^{\infty}(\Omega)}+\gamma\left\|v_{\varepsilon}\right\|_{L^{\infty}(\Omega)}\left\|w_{\varepsilon}\right\|_{L^{\infty}(\Omega)}|\Omega|
\end{aligned}
$$

and that therefore

$$
\begin{aligned}
\int_{0}^{T}\left\|w_{\varepsilon t}(\cdot, t)\right\|_{\left(W_{0}^{1, \infty}(\Omega)\right)^{\star}} d t \leq & \int_{0}^{T} \int_{\Omega}\left|\nabla w_{\varepsilon}\right|^{4}+|\Omega| T \\
& +\beta \cdot\left\{\int_{\Omega} u_{0}+\frac{1}{\beta} \int_{\Omega} w_{0}\right\} \cdot\left\|w_{0}\right\|_{L^{\infty}(\Omega)} \cdot T \\
& +\gamma\left\|v_{0}\right\|_{L^{\infty}(\Omega)} e^{\frac{\alpha}{\kappa}\left\|w_{0}\right\|_{L^{\infty}(\Omega)}} \cdot\left\|w_{0}\right\|_{L^{\infty}(\Omega)} \cdot|\Omega| T \quad \text { for all } \varepsilon \in(0,1)
\end{aligned}
$$

thanks to (2.7), (2.9) and (2.8). According to (2.23) and (2.11), this hence entails (2.31).

Our collection of estimates now enables us to pass to the limit in the following sense: 
Lemma 2.10 Let $n \leq 5$, and suppose that $u_{0}, v_{0}$ and $w_{0}$ satisfy (1.4) and (1.5). Then there exist $\left(\varepsilon_{j}\right)_{j \in \mathbb{N}} \subset(0,1)$ and nonnegative functions

$$
\left\{\begin{array}{l}
u \in L^{\infty}\left((0, \infty) ; L^{1}(\Omega)\right) \cap L_{l o c}^{\frac{n+2}{n}}\left([0, \infty) ; L^{\frac{n+2}{n}}(\Omega)\right) \cap L_{l o c}^{\frac{n+2}{n+1}}\left([0, \infty) ; W^{1, \frac{n+2}{n+1}}(\Omega)\right), \\
v \in L^{\infty}(\Omega \times(0, \infty)) \quad \text { and } \\
w \in L^{\infty}(\Omega \times(0, \infty)) \cap L_{l o c}^{4}\left([0, \infty) ; W^{1,4}(\Omega)\right) \cap L_{l o c}^{2}\left([0, \infty) ; W^{2,2}(\Omega)\right)
\end{array}\right.
$$

such that $\varepsilon_{j} \searrow 0$ as $j \rightarrow \infty$ and that

$$
\begin{aligned}
& u_{\varepsilon} \rightarrow u \quad \text { a.e. in } \Omega \times(0, \infty), \\
& u_{\varepsilon} \rightarrow u, F_{\varepsilon}\left(u_{\varepsilon}\right) \rightarrow u \quad \text { and } \quad u_{\varepsilon} F_{\varepsilon}^{\prime}\left(u_{\varepsilon}\right) \rightarrow u \quad \text { in } L_{l o c}^{p}(\bar{\Omega} \times[0, \infty)) \quad \text { for all } p \in\left[1, \frac{n+2}{n}\right), \\
& \nabla u_{\varepsilon} \rightarrow \nabla u \quad \text { in } L_{l o c}^{\frac{n+2}{n+1}}(\bar{\Omega} \times[0, \infty)), \\
& u_{\varepsilon t} \rightarrow u_{t} \quad \text { in } L_{l o c}^{q}\left([0, \infty) ;\left(W^{1, p}(\Omega)\right)^{\star}\right) \quad \text { for } p \text { and } q \text { as in (2.30), } \\
& v_{\varepsilon} \rightarrow v \quad \text { a.e. in } \Omega \times(0, \infty) \quad \text { and } \quad v_{\varepsilon}(\cdot, t) \rightarrow v(\cdot, t) \quad \text { a.e. in } \Omega \text { for a.e. } t>0, \\
& w_{\varepsilon} \rightarrow w \quad \text { a.e. in } \Omega \times(0, \infty), \\
& \nabla w_{\varepsilon} \rightarrow \nabla w \quad \text { in } L_{l o c}^{4}(\bar{\Omega} \times[0, \infty)) \quad \text { and } \\
& \Delta w_{\varepsilon} \rightarrow \Delta w \quad \text { in } L_{l o c}^{2}(\bar{\Omega} \times[0, \infty))
\end{aligned}
$$

as $\varepsilon=\varepsilon_{j} \searrow 0$. Moreover, $(u, v, w)$ is a global weak solution of (1.3) in the sense of Definition 2.1.

Proof.In view of the estimates obtained in Lemmata 2.5, 2.6, 2.7, 2.8 and 2.9, this can be achieved by means of essentially straightforward extraction procedures based on the Aubin-Lions lemma and the Vitali convergence theorem; a corresponding reasoning in a closely related situation can be found in [17].

As a by-product, we immediately obtain our main result on global solvability in the original problem (1.3).

Proof of Theorem 1.1. This directly results from Lemma 2.10,

\section{Migration-driven benefit}

In order to derive the qualitative properties claimed in Theorem 1.2, let us first draw some essentially evident consequences of our estimates gained above.

Lemma 3.1 Assume (1.4). Then for all $M>0, K>0$ and $T>0$ there exists $C(M, K, T)>0$ with the property that whenever $w_{0}$ satisfies (1.5), (1.7) and (2.11), the global weak solution $(u, v, w)$ of (1.3) from Lemma 2.10 has the properties that

$$
\int_{0}^{T} \int_{\Omega} u^{\frac{n+2}{n}} \leq C(M, K, T)
$$

and

$$
\int_{0}^{T} \int_{\Omega}|\nabla u|^{\frac{n+2}{n+1}} \leq C(M, K, T)
$$


as well as

$$
\int_{0}^{T} \int_{\Omega}|\nabla w|^{4} \leq C(M, K, T)
$$

and

$$
\int_{0}^{T}\left\|u_{t}(\cdot, t)\right\|_{\left(W^{1, p}(\Omega)\right)^{\star}}^{q} d t \leq C(M, K, T)
$$

with $p>1$ and $q>1$ taken from (2.30).

Proof.In view of (2.33) and Fatou's lemma, the estimate in (3.1) directly results from (2.25). Likewise, $(3.2),(3.3)$ and (3.4) are consequences of (2.26), (2.23) and (2.29) when combined with (2.33), (2.33) and (2.33) by means of a standard argument based on lower semicontinuity with respect to weak convergence.

\subsection{Detecting a genuine Lyapunov functional}

Now a key toward our derivation of Theorem 1.2 will consist in the detection of another Lyapunovtype preoperty of (2.5) which, unlike that from Lemma 2.4. will become manifest in a genuine energy inequality. This will be derived in Lemma 3.3 on adequately combining the following simple observations.

Lemma 3.2 Suppose that (1.4) and (1.5) hold. Then for all $\varepsilon \in(0,1)$,

$$
-\frac{d}{d t} \int_{\Omega} \ln u_{\varepsilon}+D \int_{\Omega} \frac{\left|\nabla u_{\varepsilon}\right|^{2}}{u_{\varepsilon}^{2}}=\chi \int_{\Omega} F_{\varepsilon}^{\prime}\left(u_{\varepsilon}\right) \nabla u_{\varepsilon} \cdot \nabla w_{\varepsilon}-\int_{\Omega} \frac{F_{\varepsilon}\left(u_{\varepsilon}\right)}{u_{\varepsilon}} \cdot w_{\varepsilon} \quad \text { for all } t>0
$$

and

$$
\frac{1}{2} \frac{d}{d t} \int_{\Omega} w_{\varepsilon}^{2}+\int_{\Omega}\left|\nabla w_{\varepsilon}\right|^{2}=-\beta \int_{\Omega} F_{\varepsilon}\left(u_{\varepsilon}\right) w_{\varepsilon}^{2}-\gamma \int_{\Omega} v_{\varepsilon} w_{\varepsilon}^{2} \quad \text { for all } t>0
$$

as well as

$$
\int_{0}^{t} \int_{\Omega}\left|\nabla w_{\varepsilon}\right|^{2} \leq \frac{1}{2} \int_{\Omega} w_{0}^{2} \quad \text { for all } t>0
$$

Proof.The identities in (3.5) and (3.6) immediately result from testing the first and the third equation in (2.5) by $\frac{1}{u_{\varepsilon}}$ and $w_{\varepsilon}$, respectively. Thereafter, (3.7) follows from (3.6) on integrating in time and dropping two favorably signed summands.

Taking suitable linear combinations of the above identities and additionally using further basic information on mass evolution in (2.5), we indeed obtain the following crucial inequality.

Lemma 3.3 Assume (1.4) and (1.5), and with $\kappa>0$ as in (2.10), let

$$
a:=\frac{\alpha+\frac{1}{4}}{\kappa} \quad \text { and } \quad b:=\frac{\chi^{2}}{4 D} .
$$

Then for all $\varepsilon \in(0,1)$,

$$
\frac{d}{d t}\left\{\int_{\Omega} \ln \frac{v_{\varepsilon}}{u_{\varepsilon}}+a \int_{\Omega} w_{\varepsilon}+b \int_{\Omega} w_{\varepsilon}^{2}\right\} \leq-\frac{D}{2} \int_{\Omega} \frac{\left|\nabla u_{\varepsilon}\right|^{2}}{u_{\varepsilon}^{2}}-\frac{1}{4} \int_{\Omega} w_{\varepsilon} \quad \text { for all } t>0 .
$$


Proof.Using (2.5), for $t>0$ we compute

$$
\frac{d}{d t} \int_{\Omega} \ln v_{\varepsilon}=\alpha \int_{\Omega} w_{\varepsilon} \quad \text { for all } t>0
$$

and

$$
\frac{d}{d t} \int_{\Omega} w_{\varepsilon}=-\beta \int_{\Omega} F_{\varepsilon}\left(u_{\varepsilon}\right) w_{\varepsilon}-\gamma \int_{\Omega} v_{\varepsilon} w_{\varepsilon} \quad \text { for all } t>0
$$

whence in view of (2.8) and (2.10) we have

$$
a \frac{d}{d t} \int_{\Omega} w_{\varepsilon} \leq-a \kappa \int_{\Omega} w_{\varepsilon} \quad \text { for all } t>0 .
$$

As moreover

$$
b \frac{d}{d t} \int_{\Omega} w_{\varepsilon}^{2} \leq-2 b \int_{\Omega}\left|\nabla w_{\varepsilon}\right|^{2} \quad \text { for all } t>0
$$

by (3.6), recalling (3.5) we obtain

$$
\begin{aligned}
\frac{d}{d t}\left\{\int_{\Omega} \ln \frac{v_{\varepsilon}}{u_{\varepsilon}}+a \int_{\Omega} w_{\varepsilon}+b \int_{\Omega} w_{\varepsilon}^{2}\right\} \leq & \alpha \int_{\Omega} w_{\varepsilon} \\
& -D \int_{\Omega} \frac{\left|\nabla u_{\varepsilon}\right|^{2}}{u_{\varepsilon}^{2}}+\chi \int_{\Omega} \frac{F_{\varepsilon}^{\prime}\left(u_{\varepsilon}\right)}{u_{\varepsilon}} \nabla u_{\varepsilon} \cdot \nabla w_{\varepsilon}-\int_{\Omega} \frac{F_{\varepsilon}\left(u_{\varepsilon}\right)}{u_{\varepsilon}} w_{\varepsilon} \\
& -a \kappa \int_{\Omega} w_{\varepsilon} \\
& -2 b \int_{\Omega}\left|\nabla w_{\varepsilon}\right|^{2} \\
\leq & -D \int_{\Omega} \frac{\left|\nabla u_{\varepsilon}\right|^{2}}{u_{\varepsilon}^{2}}+\chi \int_{\Omega} \frac{F_{\varepsilon}^{\prime}\left(u_{\varepsilon}\right)}{u_{\varepsilon}} \nabla u_{\varepsilon} \cdot \nabla w_{\varepsilon} \\
& -2 b \int_{\Omega}\left|\nabla w_{\varepsilon}\right|^{2}-\frac{1}{4} \int_{\Omega} w_{\varepsilon} \quad \text { for all } t>0,
\end{aligned}
$$

because $F_{\varepsilon} \geq 0$ by (2.4), and because $\alpha-a \kappa=-\frac{1}{4}$ due to (3.8). Here we use Young's inequality and again (2.4) to estimate

$$
\begin{aligned}
\chi \int_{\Omega} \frac{F_{\varepsilon}^{\prime}\left(u_{\varepsilon}\right)}{u_{\varepsilon}} \nabla u_{\varepsilon} \cdot \nabla w_{\varepsilon} & \leq \frac{D}{2} \int_{\Omega} \frac{\left|\nabla u_{\varepsilon}\right|^{2}}{u_{\varepsilon}^{2}}+\frac{\chi^{2}}{2 D} \int_{\Omega} F_{\varepsilon}^{\prime 2}\left(u_{\varepsilon}\right)\left|\nabla w_{\varepsilon}\right|^{2} \\
& \leq \frac{D}{2} \int_{\Omega} \frac{\left|\nabla u_{\varepsilon}\right|^{2}}{u_{\varepsilon}^{2}}+\frac{\chi^{2}}{2 D} \int_{\Omega}\left|\nabla w_{\varepsilon}\right|^{2} \quad \text { for all } t>0
\end{aligned}
$$

so that (3.9) becomes a consequence of (3.10) and the fact that $\frac{\chi^{2}}{2 D}=2 b$ by (3.8).

By integration, this immediately implies the following. 
Lemma 3.4 Assume (1.4) and (1.5), and let $a>0$ and $b>0$ be as in (3.8). Then for all $\varepsilon \in(0,1)$,

$$
\int_{\Omega} \ln \frac{v_{\varepsilon}(\cdot, t)}{u_{\varepsilon}(\cdot, t)}+\frac{D}{2} \int_{0}^{t} \int_{\Omega} \frac{\left|\nabla u_{\varepsilon}\right|^{2}}{u_{\varepsilon}^{2}}+\frac{1}{4} \int_{0}^{t} \int_{\Omega} w_{\varepsilon} \leq \int_{\Omega} \ln \frac{v_{0}}{u_{0}}+a \int_{\Omega} w_{0}+b \int_{\Omega} w_{0}^{2} \quad \text { for all } t>0 .
$$

In particular, whenever (1.4) holds and $M>0$ and $K>0$ are given, one can find $C(M, K)>0$ such that if $w_{0}$ satisfies (1.5), (1.7) and (2.11), then for all $\varepsilon \in(0,1)$,

$$
\int_{\Omega} \ln u_{\varepsilon}(\cdot, t) \geq-C(M, K) \quad \text { for all } t>0
$$

and

$$
\int_{0}^{\infty} \int_{\Omega} \frac{\left|\nabla u_{\varepsilon}\right|^{2}}{u_{\varepsilon}^{2}} \leq C(M, K)
$$

Proof.According to Lemma 3.3, we have

$$
\frac{d}{d t}\left\{\int_{\Omega} \ln \frac{v_{\varepsilon}}{u_{\varepsilon}}+a \int_{\Omega} w_{\varepsilon}+b \int_{\Omega} w_{\varepsilon}^{2}\right\} \leq-\frac{D}{4} \int_{\Omega} \frac{\left|\nabla u_{\varepsilon}\right|^{2}}{u_{\varepsilon}^{2}} \quad \text { for all } t>0
$$

from which (3.11) and hence also (3.12) and (3.13) immediately result upon integration.

\subsection{Estimating migration effects from below. Proof of Theorem 1.2}

It will now be of fundamental importance to make sure that the dissipative action expressed through the appearance of the second summand on the left of (3.11) is conveniently substantial. At the level of approximate solutions, a preparation for this can be gained by once again going back to (3.5):

Lemma 3.5 Assume (1.4) and (1.5). Then for all $\varepsilon \in(0,1)$,

$$
2 D \int_{0}^{t_{\star}} \int_{\Omega} \frac{\left|\nabla u_{\varepsilon}\right|^{2}}{u_{\varepsilon}^{2}} \geq \int_{\Omega} \ln u_{\varepsilon}\left(\cdot, t_{\star}\right)-\int_{\Omega} \ln u_{0}-\frac{\chi^{2}}{8 D} \int_{\Omega} w_{0}^{2}-\int_{0}^{t_{\star}} \int_{\Omega} w_{\varepsilon} \quad \text { for all } t_{\star}>0 .
$$

Proof.Again starting from (3.5), we now estimate the first summand on the right-hand side therein from below by using Young's inequality and (2.4) according to

$$
\begin{aligned}
\chi \int_{\Omega} \frac{F_{\varepsilon}^{\prime}\left(u_{\varepsilon}\right)}{u_{\varepsilon}} \nabla u_{\varepsilon} \cdot \nabla w_{\varepsilon} & \geq-D \int_{\Omega} \frac{\left|\nabla u_{\varepsilon}\right|^{2}}{u_{\varepsilon}^{2}}-\frac{\chi^{2}}{4 D} \int_{\Omega} F_{\varepsilon}^{\prime 2}\left(u_{\varepsilon}\right)\left|\nabla w_{\varepsilon}\right|^{2} \\
& \geq-D \int_{\Omega} \frac{\left|\nabla u_{\varepsilon}\right|^{2}}{u_{\varepsilon}^{2}}-\frac{\chi^{2}}{4 D} \int_{\Omega}\left|\nabla w_{\varepsilon}\right|^{2} \quad \text { for all } t>0,
\end{aligned}
$$

whence integrating (3.5) in time we obtain

$2 D \int_{0}^{t_{\star}} \int_{\Omega} \frac{\left|\nabla u_{\varepsilon}\right|^{2}}{u_{\varepsilon}^{2}} \geq \int_{\Omega} \ln u_{\varepsilon}\left(\cdot, t_{\star}\right)-\int_{\Omega} \ln u_{0}-\frac{\chi^{2}}{4 D} \int_{0}^{t_{\star}} \int_{\Omega}\left|\nabla w_{\varepsilon}\right|^{2}-\int_{0}^{t_{\star}} \int_{\Omega} \frac{F_{\varepsilon}\left(u_{\varepsilon}\right)}{u_{\varepsilon}} w_{\varepsilon} \quad$ for all $t_{\star}>0$.

As $\frac{F_{\varepsilon}\left(u_{\varepsilon}\right)}{u_{\varepsilon}} \leq 1$ by (2.4) , in view of (3.7) this entails (3.14).

In order to appropriately pass to the limit $\varepsilon \searrow 0$ in the latter inequalities, let us make sure that also the potentially singular quantity $\ln u_{\varepsilon}$ appearing therein exhibits a favorably controlable behavior when $\varepsilon$ becomes small. This will once more be achieved by means of an Aubin-Lions type argument on the basis of the following additional regularity property. 
Lemma 3.6 If (1.4) holds, then for all $M>0, K>0$ and $T>0$ one can find $C(M, K, T)>0$ such that whenever $w_{0}$ satisfies (1.5), (1.7) and (2.11), we have

$$
\int_{0}^{T}\left\|\partial_{t} \ln u_{\varepsilon}(\cdot, t)\right\|_{\left(W_{0}^{1, \infty}(\Omega)\right)^{\star}} d t \leq C(M, K, T) \quad \text { for all } \varepsilon \in(0,1) .
$$

Proof.For $\psi \in C_{0}^{\infty}(\Omega)$ fulfilling $\|\psi\|_{W^{1, \infty}(\Omega)} \leq 1$, from the first equation in (2.5) we see that for all $t>0$ and $\varepsilon \in(0,1)$,

$$
\begin{aligned}
\left|\int_{\Omega} \partial_{t} \ln u_{\varepsilon}(\cdot, t) \cdot \psi\right|= & \left|\int_{\Omega} \frac{1}{u_{\varepsilon}} \cdot\left\{D \Delta u_{\varepsilon}-\chi \nabla \cdot\left(u_{\varepsilon} F_{\varepsilon}^{\prime}\left(u_{\varepsilon}\right) \nabla w_{\varepsilon}\right)+F_{\varepsilon}\left(u_{\varepsilon}\right) w_{\varepsilon}\right\} \cdot \psi\right| \\
= & \mid D \int_{\Omega} \frac{\left|\nabla u_{\varepsilon}\right|^{2}}{u_{\varepsilon}^{2}} \psi-D \int_{\Omega} \frac{1}{u_{\varepsilon}} \nabla u_{\varepsilon} \cdot \nabla \psi \\
& \quad-\chi \int_{\Omega} \frac{F_{\varepsilon}^{\prime}\left(u_{\varepsilon}\right)}{u_{\varepsilon}}\left(\nabla u_{\varepsilon} \cdot \nabla w_{\varepsilon}\right) \psi+\chi \int_{\Omega} F_{\varepsilon}^{\prime}\left(u_{\varepsilon}\right) \nabla w_{\varepsilon} \cdot \nabla \psi+\int_{\Omega} \frac{F_{\varepsilon}\left(u_{\varepsilon}\right)}{u_{\varepsilon}} w_{\varepsilon} \psi \mid \\
\leq & D \int_{\Omega} \frac{\left|\nabla u_{\varepsilon}\right|^{2}}{u_{\varepsilon}^{2}}+D \int_{\Omega} \frac{\left|\nabla u_{\varepsilon}\right|}{u_{\varepsilon}}+\chi \int_{\Omega} \frac{\left|\nabla u_{\varepsilon}\right|}{u_{\varepsilon}} \cdot\left|\nabla w_{\varepsilon}\right|+\chi \int_{\Omega}\left|\nabla w_{\varepsilon}\right|+\int_{\Omega} w_{\varepsilon} \\
\leq & D \int_{\Omega} \frac{\left|\nabla u_{\varepsilon}\right|^{2}}{u_{\varepsilon}^{2}}+\frac{D}{2} \int_{\Omega} \frac{\left|\nabla u_{\varepsilon}\right|^{2}}{u_{\varepsilon}^{2}}+\frac{D|\Omega|}{2} \\
& +\frac{\chi}{2} \int_{\Omega} \frac{\left|\nabla u_{\varepsilon}\right|^{2}}{u_{\varepsilon}^{2}}+\frac{\chi}{2} \int_{\Omega}\left|\nabla w_{\varepsilon}\right|^{2}+\frac{\chi}{2} \int_{\Omega}\left|\nabla w_{\varepsilon}\right|^{2}+\frac{\chi|\Omega|}{2}+|\Omega| \cdot\left\|w_{\varepsilon}\right\|_{L^{\infty}(\Omega)}
\end{aligned}
$$

according to (2.4) and Young's inequality. Thus, for all $t>0$ and $\varepsilon \in(0,1)$ we have

$$
\left\|\partial_{t} \ln u_{\varepsilon}(\cdot, t)\right\|_{\left(W_{0}^{1, \infty}(\Omega)\right)^{\star}} \leq \frac{3 D+\chi}{2} \int_{\Omega} \frac{\left|\nabla u_{\varepsilon}\right|^{2}}{u_{\varepsilon}^{2}}+\chi \int_{\Omega}\left|\nabla w_{\varepsilon}\right|^{2}+|\Omega| \cdot\left\|w_{\varepsilon}\right\|_{L^{\infty}(\Omega)}+\frac{(D+\chi)|\Omega|}{2},
$$

which by an integration results in (3.15) due to (3.13), (3.7) and (2.9).

Indeed, we therefore obtain the following.

Lemma 3.7 i) Assume that (1.4) and (1.5) hold, and let $\left(\varepsilon_{j}\right)_{j \in \mathbb{N}}$ be as in Lemma [2.10. Then $\ln u \in L^{1}(\Omega \times(0, \infty))$, and there exist a null set $N \subset(0, \infty)$ and a subsequence $\left(\varepsilon_{j_{l}}\right)_{l \in \mathbb{N}}$ of $\left(\varepsilon_{j}\right)_{j \in \mathbb{N}}$ such that

$$
\ln u(\cdot, t) \in L^{1}(\Omega) \quad \text { for all } t \in(0, \infty) \backslash N
$$

and

$$
\int_{\Omega} \ln u_{\varepsilon}(\cdot, t) \rightarrow \int_{\Omega} \ln u(\cdot, t) \quad \text { for all } t \in(0, \infty) \backslash N
$$

as $\varepsilon=\varepsilon_{j_{l}} \searrow 0$.

ii) Assume (1.4), and suppose that $\left(w_{0 k}\right)_{k \in \mathbb{N}} \subset W^{1, \infty}(\Omega)$ is such that $w_{0 k} \geq 0$ in $\Omega$ as well as $\sqrt{w_{0 k}} \in W^{1,2}(\Omega)$ for all $k \in \mathbb{N}$ and that

$$
\sup _{k \in \mathbb{N}}\left\|w_{0 k}\right\|_{L^{\infty}(\Omega)}<\infty \quad \text { and } \quad \sup _{k \in \mathbb{N}} \int_{\Omega} \frac{\left|\nabla w_{0 k}\right|^{2}}{w_{0 k}}<\infty .
$$

Then the corrseponding global weak solutions $\left(u_{k}, v_{k}, w_{k}\right)$ obtained in Theorem 1.1 with initial data $\left(u_{0}, v_{0}, w_{0 k}\right)$ have the property that $\ln u_{k} \in L_{l o c}^{1}(\bar{\Omega} \times[0, \infty))$ for all $k \in \mathbb{N}$ and that $\left(\ln u_{k}\right)_{k \in \mathbb{N}}$ is relatively compact in $L_{l o c}^{1}(\bar{\Omega} \times[0, \infty))$. 
Proof.i) Since

$$
\begin{aligned}
\int_{\Omega}\left|\ln u_{\varepsilon}(\cdot, t)\right| & =2 \int_{\left\{u_{\varepsilon}(\cdot, t) \geq 1\right\}} \ln u_{\varepsilon}(\cdot, t)-\int_{\Omega} \ln u_{\varepsilon}(\cdot, t) \\
& \leq 2 \int_{\left\{u_{\varepsilon}(\cdot, t) \geq 1\right\}} u_{\varepsilon}(\cdot, t)-\int_{\Omega} \ln u_{\varepsilon}(\cdot, t) \\
& \leq 2 \cdot\left\{\int_{\Omega} u_{0}+\frac{1}{\beta} \int_{\Omega} w_{0}\right\}-\int_{\Omega} \ln u_{\varepsilon}(\cdot, t) \quad \text { for all } t>0 \text { and } \varepsilon \in(0,1)
\end{aligned}
$$

by (2.7), it readily follows from (3.12), (3.13) and standard $L^{1}-L^{2}$ interpolation based on the Gagliardo-Nirenberg inequality that for each $T>0,\left(\ln u_{\varepsilon}\right)_{\varepsilon \in(0,1)}$ is bounded in $L^{2}\left((0, T) ; W^{1,2}(\Omega)\right)$. As Lemma 3.6 asserts that moreover $\left(\partial_{t} \ln u_{\varepsilon}\right)_{\varepsilon \in(0,1)}$ is bounded in $L^{1}\left((0, T) ;\left(W_{0}^{1, \infty}(\Omega)\right)^{\star}\right)$ for any such $T$, an Aubin-Lions lemma warrants strong precompactness of $\left(\ln u_{\varepsilon}\right)_{\varepsilon \in(0,1)}$ in $L_{l o c}^{1}(\bar{\Omega} \times[0, \infty))$, implying that for a suitable subsequence $\left(\varepsilon_{j_{l}}\right)_{l \in \mathbb{N}}$ of $\left(\varepsilon_{j}\right)_{j \in \mathbb{N}}$ we can achieve that

$$
\ln u_{\varepsilon} \rightarrow z \quad \text { in } L_{l o c}^{1}(\bar{\Omega} \times[0, \infty)) \text { and a.e. in } \Omega \times(0, \infty)
$$

and

$$
\ln u_{\varepsilon}(\cdot, t) \rightarrow z(\cdot, t) \quad \text { in } L^{1}(\Omega) \quad \text { for a.e. } t>0
$$

as $\varepsilon=\varepsilon_{j_{l}} \searrow 0$. Since (3.20) together with (2.33) requires that $z=u$ a.e. in $\Omega \times(0, \infty)$, both the inclusion $\ln u \in L_{l o c}^{1}(\bar{\Omega} \times[0, \infty))$ and (3.17) become consequences of (3.19) and (3.20) if $N \subset(0, \infty)$ is chosen appropriately.

ii) As (3.18) warrants that we can achieve independence of the estimates in Lemma 3.4 and Lemma 3.6 from $k \in \mathbb{N}$, both properties can by verified by essentially repeating the argument from i).

When applied to the inequalities from Lemma 3.4 and Lemma 3.5, this implies an upper bound for the expression $\int_{\Omega} \ln \frac{u}{v}$ involving, inter alia, the difference $\int_{\Omega} \ln u-\int_{\Omega} \ln u_{0}$ when evaluated at an arbitrary but fixed time $t_{\star}$ :

Lemma 3.8 Suppose that (1.4) and (1.5) hold, and let $a>0$ and $b>0$ be as in (3.8). Then there exists a null set $N \subset(0, \infty)$ such that

$$
\ln \frac{v(\cdot, t)}{u(\cdot, t)} \in L^{1}(\Omega) \quad \text { for all } t \in(0, \infty) \backslash N
$$

and

$$
\int_{\Omega} \ln \frac{v(\cdot, t)}{u(\cdot, t)} \leq \int_{\Omega} \ln \frac{v_{0}}{u_{0}}+a \int_{\Omega} w_{0}+\left(b+\frac{\chi^{2}}{32 D}\right) \int_{\Omega} w_{0}^{2}-\frac{1}{4}\left\{\int_{\Omega} \ln u\left(\cdot, t_{\star}\right)-\int_{\Omega} \ln u_{0}\right\}
$$

for all $t_{\star} \in(0, \infty) \backslash N$ and any $t \in\left(t_{\star}, \infty\right) \backslash N$. 
Proof.On combining Lemma 3.4 with Lemma 3.5, we obtain that whenever $t_{\star}>0$ and $t>t_{\star}$,

$$
\begin{aligned}
\int_{\Omega} \ln \frac{v_{\varepsilon}(\cdot, t)}{u_{\varepsilon}(\cdot, t)} \leq & \int_{\Omega} \ln \frac{v_{0}}{u_{0}}+a \int_{\Omega} w_{0}+b \int_{\Omega} w_{0}^{2}-\frac{D}{2} \int_{0}^{t} \int_{\Omega} \frac{\left|\nabla u_{\varepsilon}\right|^{2}}{u_{\varepsilon}^{2}}-\frac{1}{4} \int_{0}^{t} \int_{\Omega} w_{\varepsilon} \\
\leq & \int_{\Omega} \ln \frac{v_{0}}{u_{0}}+a \int_{\Omega} w_{0}+b \int_{\Omega} w_{0}^{2}-\frac{D}{2} \int_{0}^{t_{\star}} \int_{\Omega} \frac{\left|\nabla u_{\varepsilon}\right|^{2}}{u_{\varepsilon}^{2}}-\frac{1}{4} \int_{0}^{t_{\star}} \int_{\Omega} w_{\varepsilon} \\
\leq & \int_{\Omega} \ln \frac{v_{0}}{u_{0}}+a \int_{\Omega} w_{0}+b \int_{\Omega} w_{0}^{2} \\
& -\frac{1}{4}\left\{\int_{\Omega} \ln u_{\varepsilon}\left(\cdot, t_{\star}\right)-\int_{\Omega} \ln u_{0}\right\}+\frac{\chi^{2}}{32 D} \int_{\Omega} w_{0}^{2}
\end{aligned}
$$

for all $\varepsilon \in(0,1)$. Now in accordance with Lemma 3.7 we can pick a null set $N_{1} \subset(0, \infty)$ and a subsequence $\left(\varepsilon_{j_{l}}\right)_{l \in \mathbb{N}}$ of the sequence $\left(\varepsilon_{j}\right)_{j \in \mathbb{N}}$ from Lemma 2.10 such that

$$
\ln u(\cdot, t) \in L^{1}(\Omega) \quad \text { for all } t \in(0, \infty) \backslash N_{1}
$$

and

$$
\int_{\Omega} \ln u_{\varepsilon}(\cdot, t) \rightarrow \int_{\Omega} \ln u(\cdot, t) \quad \text { for all } t \in(0, \infty) \backslash N_{1} \quad \text { as } \varepsilon=\varepsilon_{j_{l}} \searrow 0 .
$$

Moreover, from Lemma 2.10] we obtain a null set $N_{2} \subset(0, \infty)$ such that as $\varepsilon=\varepsilon_{j} \searrow 0$ we have $v_{\varepsilon}(\cdot, t) \rightarrow v(\cdot, t)$ a.e. in $\Omega$ for all $t \in(0, \infty) \backslash N_{2}$. Since Lemma 2.3 says that with some $c_{1}>0$ and $c_{2}>0$

$$
c_{1} \leq v_{\varepsilon}(x, t) \leq c_{2} \quad \text { for all }(x, t) \in \Omega \times(0, \infty) \text { and each } \varepsilon \in(0,1),
$$

due to the dominated convergence theorem this entails that

$$
\ln v(\cdot, t) \in L^{1}(\Omega) \quad \text { for all } t \in(0, \infty) \backslash N_{2}
$$

and

$$
\int_{\Omega} \ln v_{\varepsilon}(\cdot, t) \rightarrow \int_{\Omega} \ln v(\cdot, t) \quad \text { for all } t \in(0, \infty) \backslash N_{2} \quad \text { as } \varepsilon=\varepsilon_{j} \searrow 0
$$

and hence, by (3.25), that if we let $N:=N_{1} \cup N_{2}$ and recall (3.24), then (3.21) holds as well as

$$
\int_{\Omega} \ln \frac{v_{\varepsilon}(\cdot, t)}{u_{\varepsilon}(\cdot, t)} \rightarrow \int_{\Omega} \ln \frac{v(\cdot, t)}{u(\cdot, t)} \quad \text { for all } t \in(0, \infty) \backslash N \quad \text { as } \varepsilon=\varepsilon_{j_{l}} \searrow 0 .
$$

Once more using (3.25), we can thus infer (3.22) from (3.23).

Now in order to suitably estimate this difference $\int_{\Omega} \ln u\left(\cdot, t_{\star}\right)-\int_{\Omega} \ln u_{0}$ from below at some $t_{\star}>0$, we shall invoke a perturbation argument which at its core resorts to a corresponding property of solutions to the heat equation.

Lemma 3.9 Suppose that $u_{0} \in C^{0}(\bar{\Omega})$ is nonnegative and such that $\int_{\Omega} \ln u_{0}>-\infty$ and $u_{0} \not \equiv$ const.. Then there exist $L>0$ and $t_{0}>0$ such that the solution $U \in C^{0}(\bar{\Omega} \times[0, \infty)) \cap C^{2,1}(\bar{\Omega} \times(0, \infty))$ of

$$
\left\{\begin{aligned}
U_{t} & =D \Delta U, & & x \in \Omega, t>0, \\
\frac{\partial U}{\partial \nu} & =0, & & x \in \partial \Omega, t>0, \\
U(x, 0) & =u_{0}(x), & & x \in \Omega,
\end{aligned}\right.
$$


satisfies $U>0$ in $\bar{\Omega} \times[0, \infty)$ and

$$
\int_{\Omega} \ln U(\cdot, t)-\int_{\Omega} \ln u_{0} \geq L \quad \text { for all } t \geq t_{0} .
$$

Proof.Since $u_{0} \not \equiv$ const., a sharp version of Jensen's inequality (see the Appendix below) states that $c_{1}:=\ln \left\{\frac{1}{|\Omega|} \int_{\Omega} u_{0}\right\}-\frac{1}{|\Omega|} \int_{\Omega} \ln u_{0}$ is positive. Using that according to a well-known stabilization property of (3.27) we have $U(\cdot, t) \rightarrow \frac{1}{|\Omega|} \int_{\Omega} u_{0}$ in $L^{\infty}(\Omega)$ as $t \rightarrow \infty$ and hence, in particular,

$$
\int_{\Omega} \ln U(\cdot, t)-\int_{\Omega} \ln u_{0} \rightarrow \int_{\Omega} \ln \left\{\frac{1}{|\Omega|} \int_{\Omega} u_{0}\right\}-\int_{\Omega} \ln u_{0}=c_{1}|\Omega| \quad \text { as } t \rightarrow \infty
$$

we readily conclude that (3.28) holds for some suitably large $t_{0}>0$ if we let, e.g., $L:=\frac{c_{1}|\Omega|}{2}$.

Indeed, the first solution components lie conveniently close to solutions of (3.27) when $w_{0}$ becomes small in an appropriate sense:

Lemma 3.10 Assume (1.4), and suppose that $\left(w_{0 k}\right)_{k \in \mathbb{N}} \subset W^{1, \infty}(\Omega)$ is such that $w_{0 k} \geq 0$ in $\Omega$ and $\sqrt{w_{0 k}} \in W^{1,2}(\Omega)$ with

$$
w_{0 k} \rightarrow 0 \quad \text { in } L^{\infty}(\Omega) \quad \text { as } k \rightarrow \infty
$$

and

$$
\sup _{k \in \mathbb{N}} \int_{\Omega} \frac{\left|\nabla w_{0 k}\right|^{2}}{w_{0 k}}<\infty
$$

Moreover, for $k \in \mathbb{N}$ let $\left(u_{k}, v_{k}, w_{k}\right)$ denote the global weak solution of (1.3) from Theorem 1.1 corresponding to $w_{0}:=w_{0 k}$, and let $U$ solve (3.27). Then there exist a null set $N \subset(0, \infty)$ and a subsequence $\left(u_{k_{l}}\right)_{l \in \mathbb{N}}$ of $\left(u_{k}\right)_{k \in \mathbb{N}}$ such that

$$
\ln u_{k_{l}}(\cdot, t) \in L^{1}(\Omega) \quad \text { for all } t \in(0, \infty) \backslash N \text { and any } l \in \mathbb{N},
$$

and that

$$
\int_{\Omega} \ln u_{k_{l}}(\cdot, t) \rightarrow \int_{\Omega} \ln U(\cdot, t) \quad \text { for all } t \in(0, \infty) \backslash N \quad \text { as } l \rightarrow \infty .
$$

Proof.According to (3.30), we may invoke Lemma 3.1 to infer from (3.1), (3.2) and (3.4) upon another application of the Aubin-Lions lemma that there exist a nonnegative function $U \in L_{l o c}^{\frac{n+2}{n+1}}\left([0, \infty) ; W^{1, \frac{n+2}{n+1}}(\Omega)\right)$ and a subsequence $\left(u_{k_{l}}\right)_{l \in \mathbb{N}}$ of $\left(u_{k}\right)_{k \in \mathbb{N}}$ such that as $l \rightarrow \infty$ we have

$$
u_{k_{l}} \rightarrow U \quad \text { in } L_{l o c}^{1}(\bar{\Omega} \times[0, \infty)) \quad \text { and } \quad \nabla u_{k_{l}} \rightarrow \nabla u \quad \text { in } L_{l o c}^{1}(\bar{\Omega} \times[0, \infty)),
$$

where in view of Lemma 3.7 ii), thanks to (3.29) and (3.30) we can also achieve on passing to a further subsequence if necessary that with some null set $N \subset(0, \infty)$ we have (3.31) as well as

$$
\int_{\Omega} \ln u_{k l}(\cdot, t) \rightarrow \int_{\Omega} \ln \widehat{U}(\cdot, t) \quad \text { for all } t \in(0, \infty) \backslash N \quad \text { as } l \rightarrow \infty .
$$

Since moreover

$$
w_{k} \rightarrow 0 \quad \text { in } L^{\infty}(\Omega \times(0, \infty)) \quad \text { as } k \rightarrow \infty
$$


by (2.9) and (3.29), it follows that if we fix any $\varphi \in C_{0}^{\infty}(\bar{\Omega} \times[0, \infty))$ additionally satisfying $\frac{\partial \varphi}{\partial \nu}=0$ on $\partial \Omega \times(0, \infty)$, then in the identity

$$
-\int_{0}^{\infty} u_{k} \varphi_{t}-\int_{\Omega} u_{0} \varphi(\cdot, 0)=-D \int_{0}^{\infty} \int_{\Omega} \nabla u_{k} \cdot \nabla \varphi+\chi \int_{0}^{\infty} \int_{\Omega} u_{k} \nabla w_{k} \cdot \nabla \varphi+\int_{0}^{\infty} \int_{\Omega} u_{k} w_{k} \varphi,
$$

by Definition 2.1 known to be valid for all $k \in \mathbb{N}$, we may choose $k=k_{l}$ and take $l \rightarrow \infty$ in the first, third and fifth summand to obtain that

$$
-\int_{0}^{\infty} \int_{\Omega} u_{k_{l}} \varphi_{t} \rightarrow-\int_{0}^{\infty} \int_{\Omega} \widehat{U} \varphi_{t}, \quad \text { and } \quad-D \int_{0}^{\infty} \int_{\Omega} \nabla u_{k_{l}} \cdot \nabla \varphi \rightarrow-D \int_{0}^{\infty} \int_{\Omega} \nabla \widehat{U} \cdot \nabla \varphi
$$

as well as

$$
\int_{0}^{\infty} \int_{\Omega} u_{k_{l}} w_{k_{l}} \varphi \rightarrow 0
$$

as $l \rightarrow \infty$. In order to show decay also of the cross-diffusive contribution to (3.36), relying on our restriction that $\frac{\partial \varphi}{\partial \nu}=0$ on $\partial \Omega \times(0, \infty)$ we once more integrate by parts therein to warrant accessibility to (3.33) and (3.35), which namely imply that indeed

$$
\chi \int_{0}^{\infty} \int_{\Omega} u_{k_{l}} \nabla w_{k_{l}} \cdot \nabla \varphi=-\chi \int_{0}^{\infty} \int_{\Omega} w_{k_{l}} \nabla u_{k_{l}} \cdot \nabla \varphi-\chi \int_{0}^{\infty} \int_{\Omega} u_{k_{l}} w_{k_{l}} \Delta \varphi \rightarrow 0 \quad \text { as } l \rightarrow \infty .
$$

Therefore, (3.36) entails that for any such $\varphi$ we have

$$
-\int_{0}^{\infty} \int_{\Omega} \widehat{U} \varphi_{t}-\int_{\Omega} u_{0} \varphi(\cdot, 0)=-D \int_{0}^{\infty} \int_{\Omega} \nabla \widehat{U} \cdot \nabla \varphi
$$

so that well-known uniqueness arguments for generalized solutions of the heat equation (e.g. reasonings proceeding by duality, as presented in [9, Proposition 52.13]) become applicable so as to assert that actually $\widehat{U}$ must coincide with $U$. The claimed approximation property (3.32) therefore results from (3.34).

In conjunction with Lemma 3.9, this shows that as $w_{0}$ becomes small in the above flavor, the quantity $\int_{\Omega} \ln u(\cdot, t)-\int_{\Omega} \ln u_{0}$ indeed remains uniformly positive within a suitable set of times:

Lemma 3.11 Assume (1.4), and let $L>0$ and $t_{0}>0$ be as in Lemma [3.9. Then for all $M>0$ there exists $\sigma_{0}(M)>0$ such that whenever (1.7) and (1.8) hold with $\sigma=\sigma_{0}(M)$, one can find a measurable set $S \subset\left(t_{0}, t_{0}+1\right)$ such that $|S| \geq \frac{1}{2}$ and $\ln u(\cdot, t) \in L^{1}(\Omega)$ for all $t \in S$ as well as

$$
\int_{\Omega} \ln u(\cdot, t)-\int_{\Omega} \ln u_{0} \geq \frac{L}{2} \quad \text { for all } t \in S
$$

Proof.In view of (3.16), we see that if the claim was false then there would exist a sequence $\left(w_{0 k}\right)_{k \in \mathbb{N}} \subset$ $W^{1, \infty}(\Omega)$ of nonnegative functions $w_{0 k}$ fulfilling $\sqrt{w_{0 k}} \in W^{1,2}(\Omega)$ and

$$
\int_{\Omega} \frac{\left|\nabla w_{0 k}\right|^{2}}{w_{0 k}} \leq M \quad \text { for all } k \in \mathbb{N}
$$


as well as

$$
w_{0 k} \rightarrow 0 \quad \text { in } L^{\infty}(\Omega) \quad \text { as } k \rightarrow \infty,
$$

but such that for all $k \in \mathbb{N}$, the measurable set

$$
S_{k}:=\left\{t \in\left(t_{0}, t_{0}+1\right) \mid \ln u(\cdot, t) \in L^{1}(\Omega) \text { with } \int_{\Omega} \ln u_{k}(\cdot, t)-\int_{\Omega} \ln u_{0}<\frac{L}{2}\right\}
$$

would satisfy

$$
\left|S_{k}\right|>\frac{1}{2}
$$

where $\left(u_{k}, v_{k}, w_{k}\right)$ denotes the associated global weak solution of (1.3) from Theorem 1.1 with $w_{0}:=$ $w_{0 k}$. Now passing to a subsequence if necessary, from Lemma 3.10 would obtain a null set $N \subset(0, \infty)$ such that (3.31) holds, and such that

$$
f_{k}(t):=\int_{\Omega} \ln u_{k}(\cdot, t)-\int_{\Omega} \ln u_{0}, \quad t \in(0, \infty) \backslash N, k \in \mathbb{N},
$$

satisfies

$$
f_{k}(t) \rightarrow f(t):=\int_{\Omega} \ln u(\cdot, t)-\int_{\Omega} \ln u_{0} \quad \text { for all } t \in(0, \infty) \backslash N \quad \text { as } k \rightarrow \infty .
$$

Since thus $f_{k} \rightarrow f$ a.e. in the bounded interval $\left(t_{0}, t_{0}+1\right)$ as $k \rightarrow \infty$, Egorov's theorem would apply so as to assert that $f_{k} \rightarrow f$ almost uniformly in this interval, in particular meaning that we could find $k_{0} \in \mathbb{N}$ and a measurable set $E \subset\left(t_{0}, t_{0}+1\right) \backslash N$ such that $|E| \geq \frac{3}{4}$ and

$$
\left|f_{k_{0}}(t)-f(t)\right| \leq \frac{L}{4} \quad \text { for all } t \in E .
$$

As thanks to Lemma 3.9 our choices of $L$ and $t_{0}$ warrant that

$$
f(t) \geq L \quad \text { for all } t \geq t_{0},
$$

this entails that

$$
f_{k_{0}}(t) \geq \frac{3 L}{4} \quad \text { for all } t \in E
$$

and that hence $E \cap S_{k_{0}}=\emptyset$. This, however, is possible only if $|E|+\left|S_{k_{0}}\right| \leq\left|\left(t_{0}, t_{0}+1\right)\right|=1$ and thus requires that $\left|S_{k_{0}}\right| \leq 1-|E| \leq \frac{1}{4}$ which is inconsistent with (3.40) and thereby proves the lemma.

Combining this with Lemma 3.8 , we finally arrive at our main result on migration-driven advantage of the first population in comparison to the static one:

Proof of Theorem 1.2. We let $\sigma_{0}(M)>0$ be as given by Lemma 3.11, and taking $a>0, b>0, L>$ 0 and $t_{0}>0$ from (3.8) and Lemma 3.9. respectively, we fix $\sigma>0$ small enough fulfilling $\sigma \leq \sigma_{0}(M)$ and

$$
a|\Omega| \sigma+\left(b+\frac{\chi^{2}}{32 D}\right)|\Omega| \sigma^{2} \leq \frac{L}{16}
$$


Thus, assuming $w_{0}$ to be compatible with (1.5), (1.7) and (1.8), from Lemma 3.8 we obtain a null set $N \subset(0, \infty)$ such that the global weak solution of (1.3) from Theorem 1.1 satisfies (3.21) and

$$
\begin{aligned}
\int_{\Omega} \ln \frac{v(\cdot, t)}{u(\cdot, t)} \leq & \int_{\Omega} \ln \frac{v_{0}}{u_{0}}+a \int_{\Omega} w_{0}+\left(b+\frac{\chi^{2}}{32 D}\right) \int_{\Omega} w_{0}^{2}-\frac{1}{4} \cdot\left\{\int_{\Omega} \ln u\left(\cdot, t_{\star}\right)-\int_{\Omega} \ln u_{0}\right\} \\
\leq & \int_{\Omega} \ln \frac{v_{0}}{u_{0}}+\frac{L}{16}-\frac{1}{4} \cdot\left\{\int_{\Omega} \ln u\left(\cdot, t_{\star}\right)-\int_{\Omega} \ln u_{0}\right\} \\
& \text { for all } t_{\star} \in(0, \infty) \backslash N \text { and any } t \in\left(t_{\star}, \infty\right) \backslash N .
\end{aligned}
$$

On the other hand, since $\sigma \leq \sigma_{0}(M)$ we may invoke Lemma 3.11 to obtain a measurable set $S \subset$ $\left(t_{0}, t_{0}+1\right) \backslash N$ such that $|S| \geq \frac{1}{2}$ and that (3.37) is valid, in particular ensuring the existence of $t_{\star} \in\left(t_{0}, t_{0}+1\right) \backslash N$ such that

$$
\frac{L}{16}-\frac{1}{4} \cdot\left\{\int_{\Omega} \ln u\left(\cdot, t_{\star}\right)-\int_{\Omega} \ln u_{0}\right\} \leq \frac{L}{16}-\frac{L}{8}=-\frac{L}{16} .
$$

Therefore, (3.41) implies that (1.9) holds if we let $C:=\frac{L}{16}$ and $T:=t_{0}+1$, whereby the proof is completed.

\section{Numerical experiments}

Below we provide results of several numerical simulations. Our aim is to get insight into behaviour of the solution to system (1.3), discussed in Theorem 1.2. In particular, we are interested in the evolution in time of $I(t)$ from (1.9) in the case which - without spatial movement - would favour species $v$, i.e. for $\alpha>\delta$. We set $D_{w}=\delta=1, \alpha=2, \beta=\gamma=200$ and manipulate only $D_{u}, \chi$, together with the initial shapes of $u_{0}, v_{0}, w_{0}>0$, observing the values of

$$
M_{*}=\int_{\Omega} \frac{\left|\nabla w_{0}\right|^{2}}{w_{0}} \quad \text { and } \quad \sigma_{*}=\left\|w_{0}\right\|_{L^{\infty}(\Omega)},
$$

i.e. the smallest possible constants in (1.7) and (1.8) in Theorem 1.2. We always choose the initial conditions in such a way that $I(0)=0$.

For numerical solution, (1.3) is discretized with a second order BDF scheme in time. On each time step we discretize the equations with third degree finite elements in space, using the FEniCS package [1].

Fixed $M_{*}=0$, varying $\sigma_{*}$. Let us choose $\Omega=[0,1] \subset R^{1}$ and $\chi=\frac{1}{2}$ and $D_{u}=20$. Let us set $u_{0}=v_{0}=\exp \left(-15\left(x-\frac{1}{2}\right)^{2}\right)$ as the initial profile 1 , that is, a Gaussian peak located in the center of $\Omega$. Assume that $w$ is constant equal to $\sigma_{*}>0$, so that $M_{*}=0$. From the left picture in Figure 1 it turns that depending on the value of $\sigma_{*}$, after sufficiently long time, $I(t)$ stabilizes either above or below zero. In particular, when there is not enough food (a situation quite common in nature, when starvation is the typical status of species), the ability to move outside occupied position certainly gives an edge and $I(t)$ remains negative throughout the simulation. On the other hand, when the faster growing species $v$ has enough food, it will finally outgrow $u$ in the sense that $I(t)>0$ for large $t$.

\footnotetext{
${ }^{1}$ These initial conditions satisfy the boundary conditions only approximately.
} 

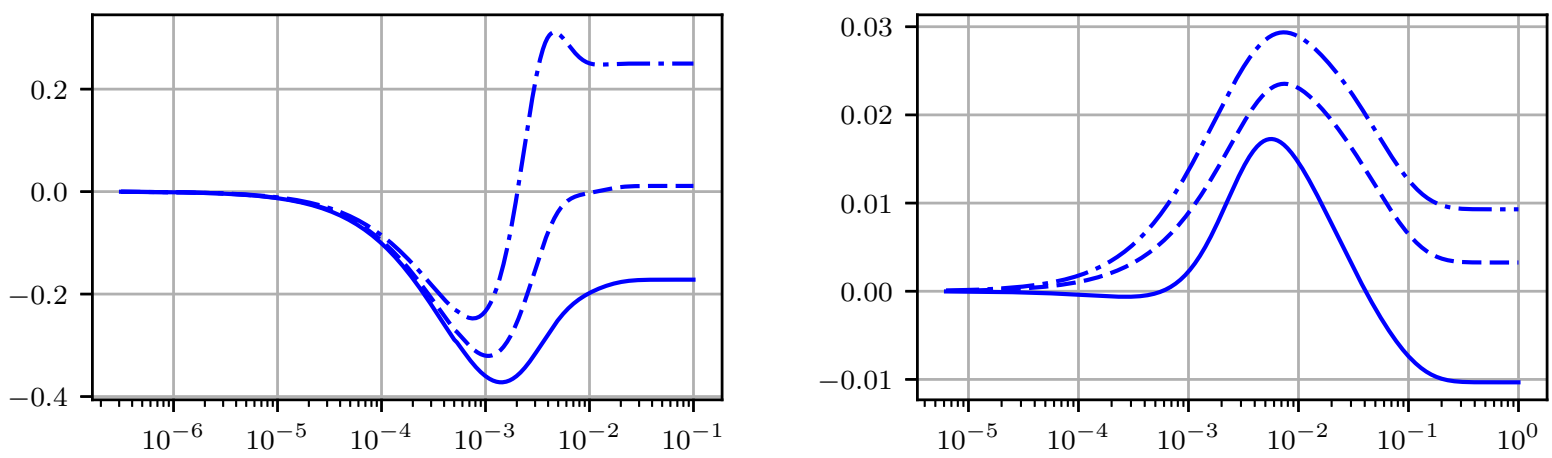

Figure 1: The evolution of $I(t)$. Left: $w_{0}(x)=\sigma_{*}$ with $\sigma_{*}$ equal to 60 (solid), 120 (dashed) or 240 (dasheddotted). Right: $w_{0}(x)=l+(20-l) \exp \left(-15\left(x-\frac{1}{2}\right)^{2}\right.$ with $l$ equal to 1.4 (solid), 14 (dashed), 20 (dashed-dotted). See the text for specification of $u_{0}$ and $v_{0}$ and other parameters, which are different for both pictures. Note the logarithmic scale of $t$.
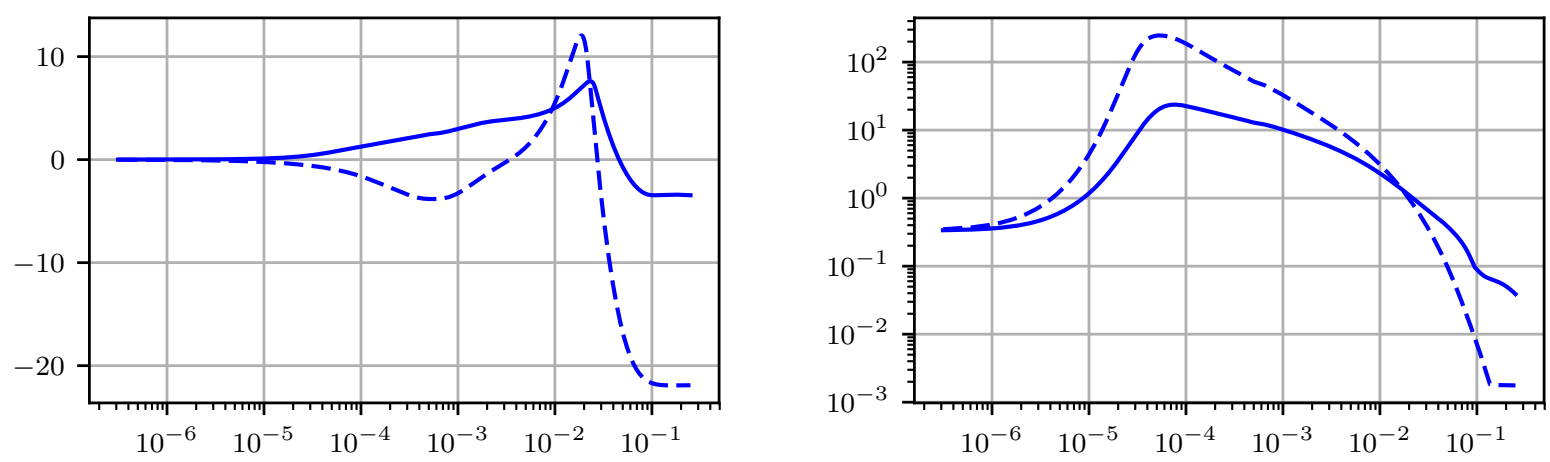

Figure 2: Behaviour of the solutions for the same initial profiles in $\Omega$ a unit ball in $R^{1}$ (solid lines) or in $R^{3}$ (dashed lines). Left: the graph of $I(t)$ with logarithmic scale of $t$. Right: the $\log -\log$ graph of $t \mapsto\left\|\frac{\partial u}{\partial r}(r, t)\right\|_{L^{\infty}(\Omega)}$.

Fixed $\sigma_{*}$, varying $M_{*}$. Next, for $D_{u}=1$ and $\chi=\frac{1}{2}$ we experiment with $u_{0}$ and $v_{0}$ with peaks at the opposite ends of $\Omega=[0,1], u_{0}(x)=1+\exp \left(-15 x^{2}\right), \quad v_{0}(x)=1+\exp \left(-15(x-1)^{2}\right)$, and the peak of food distribution $w_{0}$ located in the middle: $w_{0}(x)=l+(20-l) \exp \left(-15\left(x-\frac{1}{2}\right)^{2}\right)$, with $l \in[0,20]$. Note that $\sigma_{*}=20$ regardless of $l$, while $l$ obviously affects the value of $M_{*}$. The right graph in Figure 1 shows the behaviour of $I(t)$ for $l=1.4,14,20$, with the corresponding values of $M_{*}$ approximately equal to $170,10,0$. Note that in this setting, smaller $M_{*}$ gives bigger advantage to the non-moving species $v$.

Let us remark that for prescribed $M_{*}$ and $\sigma_{*}$, various types of behaviour of $I(t)$ are possible, depending on the shape of the initial profiles, so these two parameters alone cannot predict the evolution of the system.

Radially symmetric solution. Finally, for $D_{u}=20$ and $\chi=10^{3}$ we solve (1.3) in a unit ball $\Omega$ in $R^{d}$, where $d \in\{1,3\}$, assuming radially symmetric initial data: $u_{0}(r)=v_{0}(r)=\frac{1}{10} \exp \left(-15 r^{2}\right)$ and 
$w_{0}(r)=2 \exp \left(-15 r^{2}\right)$, where $r$ denotes the distance from the origin. It follows from Figure 2 that the solution in $R^{3}$ admits larger gradients than the corresponding solution in $R^{1}$. While in both cases $I(t)$ stabilizes on the negative side, it attains a lower level in $R^{3}$, leading to an intuition that in "larger" space the $u$ species has more space to move to, and to grow.

\section{Appendix}

\subsection{Migration-free dynamics: Decisive role of reproduction rates for ODE asymp- totics}

In order to substantiate our discussion around (1.2), let us briefly address the ODE system related to (1.1), as given by

$$
\left\{\begin{aligned}
u_{t} & =\delta u w, & & t>0 \\
v_{t} & =\alpha v w, & & t>0 \\
w_{t} & =-\beta u w-\gamma v w, & & t>0
\end{aligned}\right.
$$

with positive parameters $\alpha, \beta, \gamma$ and $\delta$, under initial conditions $u(0)=u_{0}, v(0)=v_{0}$ and $w(0)=w_{0}$.

Proposition 5.1 If $u_{0}, v_{0}$ and $w_{0}$ are positive, then (5.1) admits a unique global positive solution $(u, v, w)$, and there exist nonnegative numbers $u_{\infty}$ and $v_{\infty}$ such that

$$
u(t) \rightarrow u_{\infty}, \quad v(t) \rightarrow v_{\infty} \quad \text { and } \quad w(t) \rightarrow 0 \quad \text { as } t \rightarrow \infty .
$$

Moreover, if $v_{0}=u_{0}$ then (1.2) holds, that is, $\operatorname{sgn}\left(u_{\infty}-v_{\infty}\right)=\operatorname{sgn}(\delta-\alpha)$.

Proof.The statements on existence, uniqueness and positivity are obvious thanks to the Picard-Lindelöf theorem and a comparison argument, because the right-hand sides of (5.1) are locally Lipschitz continuous with respect to $(u, v, w)$, and because, as can easily be seen, the identity $\frac{\beta}{\delta} u+\frac{\gamma}{\alpha} v+w \equiv$ $\frac{\beta}{\delta} u_{0}+\frac{\gamma}{\alpha} v_{0}+w_{0}$ holds as long as the solution exists. Since by positivity it is clear from (5.1) that $u, v$ and $-w$ are nondecreasing we deduce (5.2). Here in the particular case when $u_{0}=v_{0}$, on separately integrating the first two equations in (5.1) we obtain that $\ln \frac{v(t)}{u(t)}=(\alpha-\delta) \int_{0}^{t} w(s) d s \geq(\alpha-\delta) \int_{0}^{1} w(s) d s$ for all $t \geq 1$, which directly shows that $u_{\infty}$ and $v_{\infty}$ have the claimed ordering property.

\subsection{A strict form of Jensen's inequality}

Since we could not find an appropriate reference, let us include a brief argument for the following essentially well-known result in which we abbreviate $\bar{\varphi}:=\frac{1}{|\Omega|} \int_{\Omega} \varphi$ for $\varphi \in L^{1}(\Omega)$.

Proposition 5.2 Let $J \subset \mathbb{R}$ be an open interval and $\Psi \in C^{2}(J)$ be strictly concave. Then

$$
\Psi(\bar{\varphi})>\overline{\Psi(\varphi)} \quad \text { for all nonconstant } \varphi \in C^{0}(\bar{\Omega} ; J)
$$

Proof.According to the Taylor theorem, for each $x \in \Omega$ one can find $\xi(x) \in J$ such that due to the strict concavity of $\Psi$,

$$
\Psi(\varphi(x))-\Psi(\bar{\varphi})-\Psi^{\prime}(\bar{\varphi}) \cdot(\varphi(x)-\bar{\varphi})=\frac{1}{2} \Psi^{\prime \prime}(\xi(x)) \cdot(\varphi(x)-\bar{\varphi})^{2} \leq-c_{1}(\varphi(x)-\bar{\varphi})^{2}
$$


with $c_{1}:=\frac{1}{2} \min _{\varphi(\bar{\Omega})}\left|\Psi^{\prime \prime}\right|>0$. After integration, this yields $\overline{\Psi(\varphi)}-\Psi(\varphi)-0 \leq c_{1} \int_{\Omega}(\varphi(x)-\bar{\varphi})^{2} d x$ and thereby implies (5.3), because $\varphi \not \equiv$ const.

\section{References}

[1] Alnæs, M.S., Blechta, J., Hake, J., Johansson, A., Kehlet, B., Logg, A., Richardson, Ch., Ring, J., and Marie E. Rognes, M.,E. N. Wells, G.,N.,: The FEniCS Project Version 1.5 Archive of Numerical Software 100 9-23 (2015)

[2] Ballyk, M., Dung, L., Jones, D.A., Smith, H.L.: Effects of random motility on microbial growth and competition in a flow reactor. SIAM J. Appl. Math. 59, 573-596 (1999)

[3] Dapsis, K., Kelly,F., Lauffenburger, D.: Effect of bacterial chemotaxis on dynamics of microbial competition. Microbial Ecology 16, 115-131 (1988)

[4] Dung, L., Smith, H.L.: A Parabolic System Modeling Microbial Competition in an Unmixed Bio-reactor. J. Differential Eq. 130, 59-91 (1996)

[5] Dung, L.: Dynamics of a bio-reactor model with chemotaxis. J. Math. Anal. Appl. 275 188-207 (2002)

[6] Hsu, S.B. : Limiting behaviour for competing species. SIAM J. Appl. Math. 34 760-763 (1978)

[7] Hsu, S.B. And Waltman, P: On a system of reaction-diffusion equations arising from competition in an unstirred Chemostat. SIAM J. Appl. Math. 53 1026-1044 (1993)

[8] Lions, P.L.: Résolution de problèmes elliptiques quasilinéaires. Arch. Rat. Mech. Anal. 74, 335-353 (1980)

[9] Quittner, P., Souplet, Ph.: Superlinear Parabolic Problems. Blow-up, Global Existence and Steady States. Birkhäuser Advanced Texts, Basel/Boston/Berlin, 2007

[10] Tans-Kersten, J., Huang, H., Allen, C.: Ralstonia solanacearum Needs Motility for Invasive Virulence on Tomato. J. Bacteriology 183, 3597-3605 (2001)

[11] TaO, Y., Winkler, M.: Eventual smoothness and stabilization of large-data solutions in a three-dimensional chemotaxis system with consumption of chemoattractant. J. Differential Eq. 252, 2520-2543 (2012)

[12] TaO, Y., Winkler, M.: Boundedness and stabilization in a multi-dimensional chemotaxishaptotaxis model. Proc. Roy. Soc. Edinburgh Sect. A 144, 1067-1084 (2014)

[13] Tilman, D.: Resource Competition and Community Structure. Princton Univeristy Press,Princton/New Jersay 1982 
[14] Turnbull, G.A., Morgan, A.W., Whipps, J. M.,Saunders, J.R.: The role of bacterial motility in the survival and spread of Pseudomonas fluorescens in soil and in the attachment and colonisation of wheat roots. FEMS Microbial Ecology 36, 21-31 (2001)

[15] Wang, X.: Qualitative behavior of solutions of a chemotactic diffusion system: Effects of motility and chemotaxis and dynamics. SIAM J. Math. Anal. 31, 535-560 (2000)

[16] Winkler, M.: Global large-data solutions in a chemotaxis-(Navier-)Stokes system modeling cellular swimming in fluid drops. Comm. Partial Differ. Eq. 37, 319-351 (2012)

[17] Winkler, M.: Asymptotic homogenization in a three-dimensional nutrient taxis system involving food-supported proliferation. J. Differential Eq. 263, 4826-4869 (2017)

[18] Yao, J And Allen, C.: Chemotaxis Is Required for Virulence and Competitive Fitness of the Bacterial Wilt Pathogen Ralstonia solanacearum. J. Bacteriology 188, 3697-3708 (2006) 